\title{
Preparation Optimization, Characterization, and Antioxidant and Prebiotic Activities of Carboxymethylated Polysaccharides from Jujube
}

\author{
Runfang Feng $\mathbb{D}^{1,2}$, Jingjing Kou $\mathbb{D}^{1,3}$ Shan Chen $\mathbb{C}^{1,3}$ Na Wang $\mathbb{D}^{1,3}$ Weiwei Wang $\mathbb{D}^{1,3}$

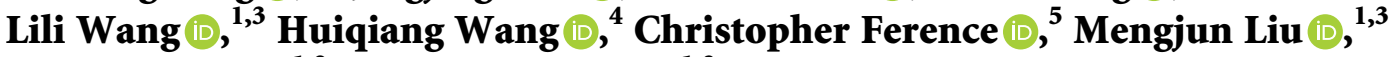 \\ Changwei Ao $\mathbb{D},,^{1,2}$ and Zhihui Zhao $\mathbb{D}^{1,3}$ \\ ${ }^{1}$ Chinese Jujube Research Center, Hebei Agricultural University, Baoding, Hebei 071000, China \\ ${ }^{2}$ College of Food Science and Technology, Hebei Agricultural University, Baoding, Hebei 071000, China \\ ${ }^{3}$ College of Horticulture, Hebei Agricultural University, Baoding, Hebei 071000, China \\ ${ }^{4}$ College of Mechanical and Electrical Engineering, Hebei Agricultural University, Baoding, Hebei 071000, China \\ ${ }^{5}$ Department of Plant Pathology, University of Florida, 2550 Hull Road, Gainesville, FL 32611, USA
}

Correspondence should be addressed to Changwei Ao; aocw@163.com and Zhihui Zhao; lyzhihuizhao@126.com

Received 11 May 2021; Revised 19 June 2021; Accepted 26 June 2021; Published 5 July 2021

Academic Editor: Shengbao Cai

Copyright ( 92021 Runfang Feng et al. This is an open access article distributed under the Creative Commons Attribution License, which permits unrestricted use, distribution, and reproduction in any medium, provided the original work is properly cited.

In this study, jujube polysaccharides (JP) were extracted from Jinsixiaozao, and carboxymethylated jujube polysaccharides (CMJP) were prepared. The optimum carboxymethylation conditions optimized by Response Surface Methodology (RSM) were as follows: the reaction temperature was $60^{\circ} \mathrm{C}$, the concentration of sodium hydroxide $(\mathrm{NaOH})$ solution was $2.8 \mathrm{~mol} / \mathrm{L}$, and the content of chloroacetic acid was $2.12 \%$ with a degree of substitution (DS) of $0.2275 \pm 0.0108$. Physicochemical characterizations and in vitro antioxidant and prebiotic activities of JP and CMJP were evaluated. Compared with unmodified JP, water solubility and viscosity were improved in CMJP. Chemical analysis revealed that CMJP was composed of Rha: Ara: Xyl: Glc: Gal=0.18: 9.09: $0.45: 0.36: 0.98$ with a molecular weight of $3.04 \times 10^{5} \mathrm{Da}$. The signals of carboxymethyl were observed at 1600,1420 , and $1328 \mathrm{~cm}^{-1}$ in FT-IR. In addition, CMJP showed obviously strong hydroxyl radical scavenging ability compared with JP and also exhibited stronger abilities than JP on the proliferation growth of Lactobacillus acidophilus, Lactobacillus plantarum, and Lactobacillus rhamnosus strains. These results indicated that CMJP could be explored as a promising resource for the development of functional foods.

\section{Introduction}

Polysaccharides are complex biopolymers comprised of monosaccharides chains and widely distributed in plants, animals, and microorganisms. As one of the most important biopolymers existing in nature, polysaccharides play diverse and important roles in many biological processes [1]. In the past two decades, natural polysaccharides have attracted great attention particularly since they can be obtained in a reproducible way from the natural sources and usually considered to have low toxicity. Natural polysaccharides have been proved to possess antitumor, antioxidant, antimicrobial, antiviral, immunomodulatory, hypoglycemic, gastrointestinal-protective, and gut microbiota-modulating properties for health promotion $[1,2]$. The biological activities of natural polysaccharide could be strongly affected by molecular modifications, such as sulfation [3], phosphorylation, carboxymethylation [4], and acetylation [5] due to the changing of their structural and conformational properties.

The application of natural polysaccharides as bioactive ingredients and food additives was limited by their complex structure and various bioactivities mechanism. Many natural polysaccharides lack useful bioactivities or exhibit weak biological activities and need to be further improved in a necessary 
way [6]. Recently, a number of studies have reported that chemical modification brings an opportunity to enhance its biological characteristics by introducing the functional groups to polysaccharides [7]. Carboxymethylation, as a versatile modification method, has been found to have great influence on the physicochemical properties and biological activities of various polysaccharides. Xu et al. [8] found that the addition of carboxymethyl groups improved the relatively poor water solubility and in vitro antioxidant activities of Ganoderma lucidum polysaccharide. Zhu et al. [9] reported that carboxymethylated Lycium barbarum polysaccharide not only enhanced the intestinal microbiota but also boosted the beneficial bacteria levels for innate immune response modulation. Carboxymethylated polysaccharides from Sargassum fusiforme also had a much higher antioxidant and antimicrobial activities in vitro than the native ones [4].

Jujube (Ziziphus jujuba Mill.) belongs to the genus Ziziphus (Rhamnaceae family) and widely distributed in the subtropical regions especially in north China (Shandong, Hebei, and Henan Provinces) [7]. Jujube fruit is rich in nutrients and functional components such as polysaccharides, vitamin C, triterpenic acid, flavonoids, and cAMP [10]. Among the several bioactive compounds present in jujube, polysaccharides, one of the most important species with a large presence in amounts, have been identified to possess antioxidant capacities [11], immunomodulation properties [12], hepatoprotective activity [13], and antitumor benefits [14], thus gaining attention as a potential pharmacological and functional supplement. Jinsixiaozao is a traditional jujube variety, which is widely planted in Cangzhou City, Hebei Province. After drying, its polysaccharide content is significantly higher than other varieties. In our earlier studies, Zhao et al. [15] isolated two pectic polysaccharides from Jinsixiaozao polysaccharides, and one of the polysaccharides was demonstrated to possess immunological activities. Moreover, recent results have revealed that the water-soluble polysaccharides extracted and purified from Jinsixiaozao polysaccharides showed antioxidant and immunomodulation activities $[16,17]$. Polysaccharides derived from Jinchangzao polysaccharides fruit represent enhanced immunomodulatory and anticoagulant activities after sulfated modification [3]. However, few researches have been carried out on the properties and the biological activities of modified especially carboxymethylated polysaccharides from Jinsixiaozao polysaccharides.

In order to further study and utilize the Jinsixiaozao polysaccharides, the aim of the present study was (i) to optimize the carboxymethylation conditions based on RSM; (ii) to analyze the preliminary physicochemical properties and structural characterizations of derivatives of carboxymethylated polysaccharides; and (iii) to evaluate the in vitro antioxidant and prebiotic activities and compare with the unmodified polysaccharides.

\section{Materials and Methods}

2.1. Materials and Reagents. The fruiting bodies of Ziziphus jujuba $c v$. Jinsixiaozao were collected from their original cultivation places located in Cangzhou, Hebei province,
China, at optimum stage of maturity and identified by one of the authors (M. J., Liu, Chinese Jujube Research Center). Samples were preserved in a cooler box $\left(0^{\circ} \mathrm{C}, 80-85 \%\right.$ relative humidity) and transported to Chinese Jujube Research Center within $3 \mathrm{~h}$. The fruit was then washed and dried to constant weight at $55^{\circ} \mathrm{C}$ through the processing line of the packaging plant. After that, kernels were removed, and fruit pulp was ground into fine powder using an electric mill (A11 basic Analytical mill, IKA-Werke Staufen, Germany) and then stored in vacuum bags at room temperature $\left(22 \pm 1^{\circ} \mathrm{C}\right)$ for later use.

Chloroacetic acid and absolute ethyl alcohol were purchased from Sinopharm Chemical Reagent Co., Ltd. (Shanghai, China). 95\% ethanol, trichloroacetic acid, concentrated hydrochloric acid $(\mathrm{HCl})$, concentrated sulfuric acid, chloroacetic acid, sodium hydroxide $(\mathrm{NaOH})$, disodium hydrogen phosphate, sodium dihydrogen phosphate, 1,1-diphenyl-2-picrylhydrazyl (DPPH), potassium sulfate, potassium ferricyanide, anhydrous iron chloride, salicylic acid, ferrous sulfate heptahydrate, hydrogen peroxide, chloroacetic acid, and vitamin $\mathrm{C}$ were purchased from Solarbio Science \& Technology Co., Ltd. (Beijing, China). Dextrans with different molecular weight (T-10, T-40, T-70, T-100, and T-500) were purchased from Solarbio Science \& Technology company (Shanghai, China). Monosaccharide standards including arabinose (Ara), rhamnose (Rha), xylose (Xyl), mannose (Man), glucose (Glc), and galactose (Gal) were obtained from Sigma Chemical Co. (St. Louis, MO, USA). All other chemicals and reagents were of analytical reagent grade. The ultrapure water was utilized from a Milli-Q water purification system (Millipore, Bedford, MA, USA).

Three bacterial strains, Lactobacillus acidophilus CGMCC 1.2919, Lactobacillus plantarum CGMCC 1.569, and Lactobacillus rhamnosus CGMCC 1.576 were obtained from the China General Microbiological Culture Collection Center (CGMCC). The strains were stored in MRS-G broth ( $2 \%$ glucose) containing $10 \%$ glycerol at $-80^{\circ} \mathrm{C}$ until use. The bacteria were activated using MRS-G broth before use.

\subsection{Preparation of JP and Its Carboxymethylated Derivatives}

2.2.1. Preparation of JP. The crude Jinsixiaozao polysaccharide (JP) was obtained according to our earlier study [15]. Briefly, dried fruit powder $(2500 \mathrm{~g})$ was immerged and washed three times with $95 \%$ ethanol $(1: 10, w / v)$ at $80^{\circ} \mathrm{C}$ for $90 \mathrm{~min}$ each time to remove some soluble materials, including free sugars, lipids, and phenols. After that, the filtrated and dried residue was extracted in boiling water $(1: 20, w / v)$ at $100^{\circ} \mathrm{C}$ for three times ( $90 \mathrm{~min}$ each time). The supernatants were combined and concentrated by rotary evaporation (IKA-WerkeRV-10, Staufen, Germany) under reduced pressure and then mixed with absolute ethanol, stirred vigorously, and kept overnight at $4^{\circ} \mathrm{C}$ to precipitate polymers. The precipitate was collected by centrifugation ( $5000 \mathrm{rpm}, 20 \mathrm{~min}$ ), deproteinated following Sevag method [18], lyophilized using a freeze-dryer (Alpha-2, Christ, Germany), and weighed, and the extraction rate of crude JP is $5.66 \%$. 
2.2.2. Preparation of Carboxymethylated Derivative. The carboxymethylation of JP was performed based on the method described by Wang et al. [19] with modifications. A solution of $\mathrm{JP}(500 \mathrm{mg})$ in $\mathrm{NaOH}(1.0,1.5,2.0,2.5,3$, and $3.5 \mathrm{~mol} / \mathrm{L}$, $100 \mathrm{~mL}$ ) was vigorously stirred with a magnet stirrer for $60 \mathrm{~min}$ at room temperature $\left(22 \pm 1^{\circ} \mathrm{C}\right)$ until a homogeneous solution was obtained. Then different content of chloroacetic acid solution $(1,2,3,4$, and 5\%) was added and stirred at a certain temperature $\left(40,50,60,70\right.$, and $\left.80^{\circ} \mathrm{C}\right)$ for $5 \mathrm{~h}$. The reaction mixture was cooled to room temperature, adjusted to $\mathrm{pH} 7.0$ with $0.5 \mathrm{~mol} / \mathrm{L}$ glacial acetic acid, and dialyzed in dialysis bags (with a molecular weight cut-off of 8000-14,000 Da) for $72 \mathrm{~h}$ with distilled water and then freeze-dried to obtain carboxymethylated jujube polysaccharide (CMJP).

The influence of the concentration of $\mathrm{NaOH}$ solution, the content of chloroacetic acid, and the reaction temperature on the degree of substitution (DS) of CMJP was studied. Singlefactor experiments showed that the optimal $\mathrm{NaOH}$ solution concentration, chloroacetic acid content, and reaction temperature were $2.5 \mathrm{~mol} / \mathrm{L}, 2 \%$, and $60^{\circ} \mathrm{C}$, respectively. On the basis of single-factor test results, response surface methodology (RSM) was used to further optimize the preparation conditions of CMJP. Three-factor three-level, Box-Behnken factorial design (BBD) was used to evaluate the combined effect of three independent variables: $\mathrm{NaOH}$ solution concentration, chloroacetic acid content, and reaction temperature, coded as $X_{1}, X_{2}$, and $X_{3}$, respectively. The DS of CMJP was regarded as the response value, and 17 experiments were required, as shown in Table S1.

The adequacy and significance in the model was evaluated by analysis of variance (ANOVA) for each response. Design-Expert 11 software package (Trial Version, Stat-Ease Inc., Minneapolis, MN, USA) was used to analyze the experimental data.

2.2.3. Determination of Degree of Substitution (DS) of CMJP. According to the neutralisation titration method, $20 \mathrm{mg}$ CMJP was dissolved in $20 \mathrm{~mL} \mathrm{NaOH}(0.01 \mathrm{~mol} / \mathrm{L})$ and stirred thoroughly with a magnet stirrer for $60 \mathrm{~min}$ at $40^{\circ} \mathrm{C}$. The solution was titrated with $0.1 \mathrm{~mol} / \mathrm{L} \mathrm{HCl}$ until the color of phenolphthalein disappeared. The DS was calculated as follows:

$$
\begin{aligned}
\mathrm{DS} & =\frac{0.162 A}{(1-0.058 A)}, \\
A & =\frac{\left[0.01 V_{1}-0.1\left(V_{2}-V_{3}\right)\right]}{W},
\end{aligned}
$$

where $A$ was the amount of $\mathrm{NaOH}$ solution consumed per gram of sample $(\mathrm{mol} / \mathrm{g}) ; V_{1}$ and $V_{2}$ were the volume of $\mathrm{NaOH}$ and $\mathrm{HCl}$ consumed, respectively $(\mathrm{mL}) ; V_{3}$ was the volume of $\mathrm{HCl}$ consumed by titrating the blank solution $(\mathrm{mL})$; and $W$ was the mass of the sample (gram).

\subsection{Characterization of Carboxymethylated Derivatives}

2.3.1. Water Solubility and Viscosity. For water solubility, $1 \mathrm{~mL}$ water was added to $100 \mathrm{mg}$ samples and then shaken vigorously for $12 \mathrm{~h}$ until the sample was completely dissolved. The precipitate was dried at $55^{\circ} \mathrm{C}$ and weighed. The rheological properties of each sample were evaluated on a rotational rheometer (Anton Paar RheolabQC model) in three replicates. $10 \mathrm{mg} / \mathrm{mL}$ aqueous solution of JP and CMJP in distilled water was prepared. A shear rate sweep from 0.1 to $1000 \mathrm{~s}^{-1}$ was applied, and rheological data were collected at $25^{\circ} \mathrm{C}$. The relationship between apparent viscosity and shear rate was modeled with a power law equation: $\eta_{a}=k Y^{(n-1)}$, where $\eta_{a}$ is apparent viscosity ( $\left.\mathrm{mPa} \mathrm{s}\right), Y$ is shear rate $\left(\mathrm{s}^{-1}\right), k$ is consistency index $(\mathrm{mPa})$, and $n$ is the flow index (dimensionless).

2.3.2. Analysis of Chemical Compositions. The total sugar contents of JP and CMJP were determined by phenol-sulfuric acid method, using $D$-glucose as the standard. The content of uronic acid was estimated using $m$-hydroxybiphenyl method with galacturonic acid as the standard.

2.3.3. Determination of the Neutral Monosaccharide Composition. JP and CMJP samples $(10 \mathrm{mg})$ were hydrolyzed with $2 \mathrm{~mol} / \mathrm{L}$ trifluoroacetic acid (TFA, $4 \mathrm{~mL}$ ) at $110^{\circ} \mathrm{C}$ for $6 \mathrm{~h}$. The hydrolyzed samples and standard monosaccharides were acetylated by the addition of hydroxylamine hydrochloride, pyridine, and acetic anhydride. The acetylated polysaccharide was analyzed by gas chromatography (GC) on an Agilent model 7890A instrument, equipped with a TG-5MS capillary column $(30 \mathrm{~m} \times 0.25 \mathrm{~mm} \times 0.25 \mu \mathrm{m})$ and a flame ionization detector (FID). The temperatures of the injector and the detector were $240^{\circ} \mathrm{C}$ and $260^{\circ} \mathrm{C}$, respectively. The flow rate of carrier gas (high-purity nitrogen, $99.999 \%$ ), air, and hydrogen were $20 \mathrm{~mL} / \mathrm{min}, 400 \mathrm{~mL} / \mathrm{min}$, and $50 \mathrm{~mL} /$ min, respectively. The nitrogen gas pressure was adjusted to $8.79 \mathrm{psi}$ at a constant linear velocity of $3 \mathrm{~cm} / \mathrm{s}$. The initial temperature was held at $140^{\circ} \mathrm{C}$ for $3 \mathrm{~min}$ and then increased at $10^{\circ} \mathrm{C} / \mathrm{min}$ to the final temperature of $240^{\circ} \mathrm{C}$ and held for $11 \mathrm{~min}$. The import of the sample into the column was accomplished using the split mode at a ratio of $20: 1$, and injection volume was $1 \mu \mathrm{L}$. The monosaccharide compositions were identified by comparing the retention time of standards and calculated by area normalization method using mass spectra of peaks on the chromatograms derived from the samples and the standard solution mix.

2.3.4. Molecular Weight (Mw) Determination. The Mw of JP and CMJP was measured using high-performance liquid chromatography (HPLC) which was undertaken on an Agilent 1200 Infinity Series LC system (Agilent Technologies, Germany) coupled with an ELSD-1260 (Agilent Technologies, UK) evaporative light detector. Samples were dissolved in ultrapure water $(0.5 \mathrm{mg} / \mathrm{mL})$, passed through a $0.22 \mu \mathrm{m}$ filter, and applied to a gel-filtration chromatographic column of Shodex KS-805 (300 $\mathrm{mm} \times 8.0 \mathrm{~mm}$, Showa Denko K.K., Japan) at $35^{\circ} \mathrm{C}$. Ultrapure water ( $\mathrm{pH} 7.0$ ) was used as the flow phase at a flow rate of $1 \mathrm{~mL} / \mathrm{min}$ with an injection volume of $20 \mu \mathrm{L}$. The column was preliminary calibrated by standard dextrans (T-500, T-100, T-70, T-40, $\mathrm{T}-10$, and glucose) with respective retention time and 
molecular weights. Then, a standard curve was plotted, and the Mw of JP and CMJP was calculated based on the standard curve.

2.3.5. Fourier Transform Infrared Spectra (FT-IR) Analysis. The infrared spectra of JP and CMJP were measured by a FTIR spectrophotometer (Spectrum 65, PerkinElmer, USA) using $\mathrm{KBr}$ compression method. One mg of JP or CMJP was ground with $200 \mathrm{mg}$ of $\mathrm{KBr}$ powder (completely dried at $100^{\circ} \mathrm{C}$ ) and pressed into a $1 \mathrm{~mm}$ agate mortar into a pellet with $1 \mathrm{~mm}$ thickness for a frequency resolution of $1 \mathrm{~cm}^{-1}$ and 32 scans between 400 and $4000 \mathrm{~cm}^{-1}$.

2.3.6. UV-Vis Spectra. Ultraviolet spectra of JP and CMJP were obtained on TU-1810 ultraviolet spectrophotometer (Purkinje General Instrument Co., Ltd., Beijing, China). The scanning range was $190-900 \mathrm{~nm}$ at $1 \mathrm{~nm}$ intervals for each sample $(0.1 \mathrm{mg} / \mathrm{mL}, \mathrm{w} / \mathrm{v}$ in distilled water $)$.

2.3.7. Scanning Electron Microscope (SEM) and EnergyDispersive X-Ray Spectroscopy (EDS) Analysis. Scanning electron micrographs of JP and CMJP were obtained with a TESCAN VEGA3 LMH scanning electron microscope (TESCAN Co., Brno, Czech Republic). Sample powders were placed on a specimen holder with the help of doublesided adhesive tapes and coated with a thin layer of gold powder. Each sample was observed with 5,000- and 10,000fold magnification at an accelerating potential of $10 \mathrm{kV}$ during micrography. Chemical analysis was performed using an energy-dispersive X-ray spectroscopy (EDS, Model TEAM, EDAX, USA) facility.

\subsection{Antioxidant Activity}

2.4.1. Scavenging Activity of DPPH Radical. The effect of scavenging DPPH radicals of JP and CMJP was determined by a reported method, with minor modification [20]. A polysaccharide solution $(1,2,3,4$, and $5 \mathrm{mg} / \mathrm{mL})$ was prepared by dissolving in distilled water. $2 \mathrm{~mL} \mathrm{DPPH}$ solution $(0.1 \mathrm{mmol}$ in ethanol) was added to $0.5 \mathrm{~mL}$ of various concentrations of sample solutions with $1.5 \mathrm{~mL}$ distilled water. The mixture was shaken rigorously and incubated in the dark for $60 \mathrm{~min}$ at room temperature; the absorbance at $517 \mathrm{~nm}$ was measured using a spectrophotometer (TU-1810, Puxi General Instrument Co., Ltd., China). Vitamin C (0.1, $0.2,0.5,1,2,3,4$, and $5 \mathrm{mg} / \mathrm{mL}$ ) was used as the positive control. DPPH radical scavenging activity of the sample was calculated as

$$
\text { DPPH scavenging rate }(\%)=\left[1-\frac{\left(A_{s}-A_{c}\right)}{A_{b}}\right] \times 100 \% \text {, }
$$

where $A_{b}$ (blank) was the absorbance of the mixture of DPPH-ethanol solution without sample; $A_{c}$ (control) was the absorbance of the mixture of ethanol and sample without
DPPH; and $A_{s}$ (sample) was the absorbance of the mixture of $\mathrm{DPPH}$ and sample.

2.4.2. Scavenging Activity of Hydroxyl radical (HO). The hydroxyl radical scavenging abilities of JP and CMJP were evaluated according to the method of Duan et al. [21] with slight modifications. $2 \mathrm{~mL}$ polysaccharide solution $(1,2,3,4$, and $5 \mathrm{mg} / \mathrm{mL}$ in distilled water) was mixed with salicylic acid $\left(0.5 \mathrm{~mL}, 9 \mathrm{mmol}\right.$ in ethanol), $\mathrm{FeSO}_{4}$ solution $(0.5 \mathrm{~mL}$, $9 \mathrm{mmol}), \mathrm{H}_{2} \mathrm{O}_{2}$ solution $(0.5 \mathrm{~mL}, 8.8 \mathrm{mmol})$, and $6.5 \mathrm{~mL}$ distilled water. The resulting solution was shaken well and incubated in a water bath at $37^{\circ} \mathrm{C}$ for $30 \mathrm{~min}$; the absorbance was determined at $510 \mathrm{~nm}$. Vitamin $\mathrm{C}(0.1,0.2,0.5,1,2,3,4$, and $5 \mathrm{mg} / \mathrm{mL}$ ) was used as the positive control. The hydroxyl radical scavenging activity was calculated as

$$
\text { HO scavenging rate }(\%)=\left[1-\frac{\left(A_{s}-A_{c}\right)}{A_{b}}\right] \times 100 \% \text {, }
$$

where $A_{b}$ (blank) was the absorbance of distilled water, $A_{c}$ (control) was the absorbance of distilled water replaced $\mathrm{FeSO}_{4}$ solution, and $A_{s}$ (sample) was the absorbance of sample.

2.4.3. Scavenging Activity of Superoxide Anion radical $\left(\mathrm{O}_{2}{ }^{-}\right)$. The effect of scavenging $\mathrm{O}_{2}{ }^{--}$of JP and CMJP was measured by the method of Liu and Huang [22] with minor modification. Different concentrations of polysaccharide solutions $(0.03125,0.0625,0.125,0.25,0.5,1,2$, and $4 \mathrm{mg} / \mathrm{mL})$ were prepared by dissolving them in distilled water. $0.5 \mathrm{~mL}$ polysaccharide solution and $3 \mathrm{~mL}$ Tris- $\mathrm{HCl}$ buffer $(50 \mathrm{mmol}$, $\mathrm{pH}$ 8.2) were mixed well and kept in a water bath at $30^{\circ} \mathrm{C}$ for $20 \mathrm{~min}$; then $3 \mathrm{~mL}$ pyrogallol solution $(7 \mathrm{mmol})$ was added and incubated for $4 \mathrm{~min}$. $1 \mathrm{~mL}$ concentrated $\mathrm{HCl}$ was added to stop the reaction, and the absorbance was measured at $420 \mathrm{~nm}$. Vitamin C $(0.1,0.2,0.5,1,2,3,4$, and $5 \mathrm{mg} / \mathrm{mL})$ was used as positive control. The superoxide anion radical scavenging activity of the sample was calculated as

$$
\mathrm{O}_{2}^{-} \text {scavenging rate }(\%)=\left[1-\frac{\left(A_{s}-A_{c}\right)}{A_{b}}\right] \times 100 \% \text {, }
$$

where $A_{b}$ (blank) was the absorbance of the blank (without sample); $A_{c}$ (control) was the absorbance of the sample without pyrogallol solution; and $A_{s}$ (sample) was the absorbance in the presence of the sample.

2.4.4. Reducing Power Assay. The reducing power of JP and CMJP was quantified by the method described by Li et al. [16] with slight modification. $1 \mathrm{~mL}$ of different concentrations of polysaccharide solution $(1,2,3,4$, and $5 \mathrm{mg} / \mathrm{mL})$, $1 \mathrm{~mL}$ potassium ferricyanide solution $(1 \%, \mathrm{w} / \mathrm{v})$, and $1 \mathrm{~mL}$ phosphate buffer $(0.2 \mathrm{~mol}, \mathrm{pH} 6.6)$ were mixed, shaken rigorously, and incubated in a water bath at $50^{\circ} \mathrm{C}$ for $20 \mathrm{~min}$. The reaction was terminated by $1 \mathrm{~mL}$ TCA solution $(10 \%, \mathrm{w} /$ v) and centrifuged at $4000 \mathrm{rpm}$ for $10 \mathrm{~min} .2 \mathrm{~mL}$ supernatant and $0.4 \mathrm{~mL}$ ferric chloride solution $(0.1 \%, w / v$ in distilled water) were reacted at room temperature for $10 \mathrm{~min}$. The 
absorbance was measured at $700 \mathrm{~nm}$. Vitamin C $(0.1,0.2,0.5$, $1,2,3,4$, and $5 \mathrm{mg} / \mathrm{mL}$ ) was used as positive control. The increasing absorbance of the reaction mixture indicated the increasing reducing power of the sample.

2.5. Prebiotic Activity Analysis. The activated Lactobacillus strains incubated in MRS medium at $37^{\circ} \mathrm{C}$ incubator for $48 \mathrm{~h}$. After centrifugation at $4^{\circ} \mathrm{C}, 5000 \mathrm{rpm}$ for $10 \mathrm{~min}$, cells were collected and adjusted to $4 \times 10^{9} \mathrm{CFU} / \mathrm{mL}$ for preparation of the growth curve. Appropriate bacterial liquid was added to the MRS medium, incubated at $37^{\circ} \mathrm{C}$ for $48 \mathrm{~h}$, and measured every $2 \mathrm{~h}$ at $600 \mathrm{~nm}$ with a spectrophotometer (TU-1810, Puxi General Instrument Co., Ltd., China). MRS broth with $2 \%(w / v)$ glucose (MRS-G, Beijing Solarbio Science \& Technology Co., Ltd., Beijing, China) was used as the basal medium to investigate the prebiotic activity in vitro and to determine whether the polysaccharides (JP and CMJP) was potential substrate to support the growth of $L$. acidophilus, L. plantarum, and L. rhamnosus. JP and CMJP were filtered and sterilized and then added to the MRS-G broth to prepare polysaccharide solutions with different contents $(0.5,1.0$, and $1.5 \%, w / v)$. The basal medium was used as a control. $2 \mu \mathrm{L}$ of active lactic acid bacteria liquid (exponential phase) was added to the MRS-G broth medium and kept in a constanttemperature incubator at $37^{\circ} \mathrm{C}$ for $24 \mathrm{~h}$, and the absorbance was measured every $6 \mathrm{~h}$. The numbers of Lactobacillus strains in the samples were obtained by measuring their optical density values at $600 \mathrm{~nm}$ with a spectrophotometer.

2.6. Statistical Analysis. Each assay of all determinations was performed in triplicate and expressed as mean \pm SD (standard deviation). One-way analysis of variance (ANOVA) followed by Duncan's multiple-range test was used for multiple comparisons by the SPSS 23.0 software package (Chicago, USA). $P<0.05$ means significant difference while $P<0.01$ means very significant difference. The results were performed with GraphPad Prism 7.0 (GraphPad Software, Inc., San Diego, CA).

\section{Results and Discussion}

3.1. Preparation of CMJP. As shown in Figure 1(a), when concentration of $\mathrm{NaOH}$ solution varied from 1 to $2.5 \mathrm{~mol} / \mathrm{L}$, the DS increased significantly and reached a maximum $(0.2249)$ at $2.5 \mathrm{~mol} / \mathrm{L}$. Once alkalization has been achieved, further increasing the $\mathrm{NaOH}$ concentration from 2.5 to $3.5 \mathrm{~mol} / \mathrm{L}$ encouraged side reactions [23]. Therefore, $2.5 \mathrm{~mol} /$ $\mathrm{L}$ was used as the optimal concentration condition for $\mathrm{NaOH}$ solution.

The DS of CMJP had been increasing when chloroacetic acid content increased from $1 \%$ to $2 \%$ as shown in Figure 1(b). The maximum DS (0.2133) of CMJP was observed when chloroacetic acid content was $2 \%$. However, at chloroacetic acid concentration greater than $2 \%, \mathrm{NaOH}$ was neutralized and further increasing the concentration of chloroacetic acid has no advantages on the modification of carboxymethylation. Therefore, $2 \%$ was used as the optimal condition for the chloroacetic acid content.
As shown in Figure 1(c), The DS of CMJP significantly increased from 0.1786 to 0.1891 as carboxymethylation temperature increased from 40 to $60^{\circ} \mathrm{C}$. However, when carboxymethylation temperature is greater than $60^{\circ} \mathrm{C}$, a significant decrease appeared which can speculate that excessively high temperature might reduce the stirring efficiency and the uniformity of the etherification reaction [24]. Therefore, $60^{\circ} \mathrm{C}$ was used as the optimal reaction temperature for the preparation of CMJP.

According to the single-parameter study, we adopted $\mathrm{NaOH}$ solution concentration of $2,2.5$, and $3 \mathrm{~mol} / \mathrm{L}$, chloroacetic acid content of $1 \%, 2 \%$, and $3 \%$, and extraction temperature of 50,60 , and $70^{\circ} \mathrm{C}$ for RSM experiments.

3.1.1. Model Fitting and Statistical Analysis. The variation of responses (DS of CMJP) at different experimental combinations was given in Table 1 . By employing multiple regression analysis on the experimental data, the predicted response $Y$ for the DS of CMJP can be obtained by the following second-order polynomial equation: $Y=-1.667+0.179 X_{1}+0.273 X_{2}$ $+0.045 X_{3}-0.001 X_{2} X_{3}-0.027 X_{1}^{2}-0.045 X_{2}^{2}$, where $X_{1}, X_{2}$, and $X_{3}$ were the coded values of the test variables, concentration of $\mathrm{NaOH}$ solution $(\mathrm{mol} / \mathrm{L})$, content of chloroacetic acid (\%), and the reaction temperature $\left({ }^{\circ} \mathrm{C}\right)$, respectively.

The $F$-value and $P$-value were used to measure the significance of the coefficients of the model. The corresponding variables would be more significant if the absolute $F$-value becomes greater and the $P$-value becomes smaller [25]. $F$-test suggested that the model had a very high $F$-value $(F=14.37)$ and a very low $P$-value $(P=0.001)$, indicating that this model was highly significant. The lack-of-fit test measures the failure of the model to represent the data in the experimental domain at points which were not included in the regression [12]. $F$-value (1.34) and $P$-value (0.3808) of lack of fit implied that it was not significant relative to the pure error, which confirmed that the model equation was adequate for predicting the DS of CMJP under any combination of values of the variables. In addition, the determined coefficient of model $\left(R^{2}=0.9487\right)$ indicated that the mode is applicable. The adjusted determination coefficient (adjusted $R^{2}=0.8826$ ) was also high, indicating a high degree of correlation between the experimental and predicted values [26]. At the same time, a low value $6.17 \%$ of coefficient of the variation (C.V.) clearly showed a very high degree of precision and a good deal of reliability of the experimental values.

The $P$-value was used as a tool to check the significance of each coefficient, which in turn may indicate the pattern of the interactions among the variables. The smaller the $P$-value was, the more significant the corresponding coefficient was [26]. As shown in Table 1, the linear coefficients $\left(X_{2}\right)$ and quadratic term coefficients $\left(X_{2}^{2}, X_{3}^{2}\right)$ were significant with very small $P$-values $(P<0.05$ or $P<0.01)$, while the other term coefficients were not significant $(P>0.05)$. Meanwhile, the content of chloroacetic acid $\left(X_{2}^{2}\right)$ was the most important 


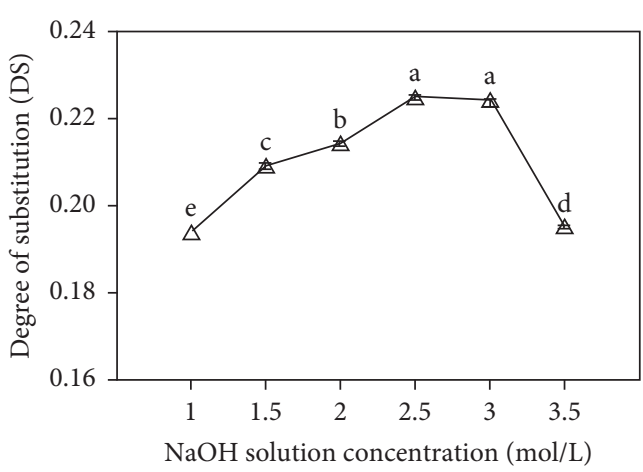

(a)

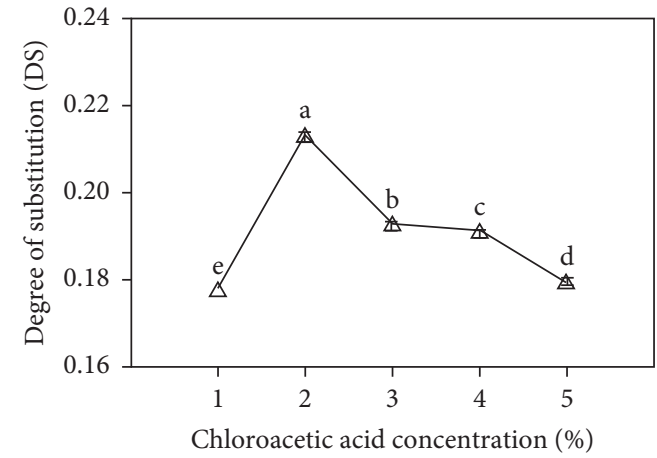

(b)

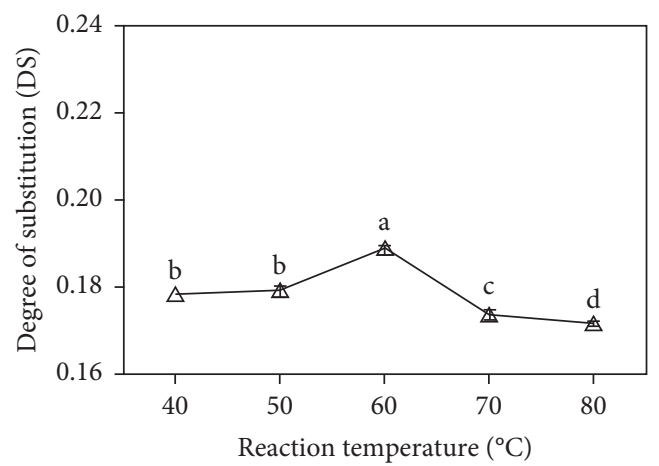

(c)

Figure 1: Effects of $\mathrm{NaOH}$ solution concentration (a), chloroacetic acid content (b), and reaction temperature (c) on the DS of CMJP. Data with different lowercase letters represent significant differences at $P<0.05$.

TABLE 1: Analysis of variance (ANOVA) for response surface quadratic model for the optimization of carboxymethylation conditions.

\begin{tabular}{|c|c|c|c|c|c|c|}
\hline Source & Sum of squares & Degree of freedom & Mean of squares & $F$-value & $P$-value & Significance \\
\hline Model & 0.0172 & 9 & 0.0019 & 14.37 & 0.0010 & $*$ \\
\hline$X_{1}$ & 0.0005 & 1 & 0.0005 & 3.90 & 0.0888 & ns \\
\hline$X_{2}$ & 0.0010 & 1 & 0.0010 & 7.59 & 0.0283 & $*$ \\
\hline$X_{3}$ & 0.0003 & 1 & 0.0003 & 1.89 & 0.2116 & ns \\
\hline$X_{1} X_{2}$ & $2.500 \mathrm{E}-09$ & 1 & $2.500 \mathrm{E}-09$ & 0.0000 & 0.9967 & ns \\
\hline$X_{1} X_{3}$ & 0.0000 & 1 & 0.0000 & 0.1519 & 0.7083 & ns \\
\hline$X_{2} X_{3}$ & 0.0007 & 1 & 0.0007 & 5.41 & 0.0530 & ns \\
\hline$X_{1}^{2}$ & 0.0002 & 1 & 0.0002 & 1.46 & 0.2655 & ns \\
\hline$X_{2}^{\frac{1}{2}}$ & 0.0087 & 1 & 0.0087 & 64.91 & $<0.0001$ & $* *$ \\
\hline$X_{3}^{2}$ & 0.0048 & 1 & 0.0048 & 36.10 & 0.0005 & $*$ \\
\hline Residual error & 0.0009 & 7 & 0.0001 & & & \\
\hline Lack of fit & 0.0005 & 3 & 0.0002 & 1.34 & 0.3808 & ns \\
\hline Pure error & 0.0005 & 4 & 0.0001 & & & \\
\hline Total & 0.0182 & 16 & & & & \\
\hline
\end{tabular}

$R^{2}=0.9487$. Adjusted $R^{2}=0.8826$. Predicted $R^{2}=0.5488$. C.V. $\%=6.17$. Adeq. precision $=12.3571 . X_{1}$ : concentration of NaOH solution, $X_{2}:$ content of chloroacetic acid solution, and $X_{3}$ : reaction temperature. ${ }^{* *}$ Extremely significant difference $(P<0.01),{ }^{*}$ significant difference $(P<0.05)$, and ns: nonsignificant.

parameter affecting the degree of carboxymethyl substitution, followed by the concentration of $\mathrm{NaOH}$ solution and the reaction temperature.

3.1.2. Optimization of Preparation Conditions of CMJP. Three-dimensional (3D) response surface and two-dimensional (2D) contour plots (Figure 2) were obtained using Design-Expert 11 to study the effects of parameters and their interactions on the maximum response. Each figure showed the effects of two factors on the DS of CMJP, while the other one was kept at zero level. The interactions between the $\mathrm{NaOH}$ concentration and the other two parameters $\left(X_{1} X_{2}\right.$ and $X_{1} X_{3}$ ) did not impact the DS of CMJP significantly. In Figure 2(e), when $\mathrm{NaOH}$ concentration $\left(X_{1}\right)$ was fixed at 0 level, chloroacetic acid content $\left(X_{2}\right)$ and reaction temperature $\left(X_{3}\right)$ demonstrated comprehensive effects on the DS. The elliptical contour plot shown in Figure 2(f) indicated 


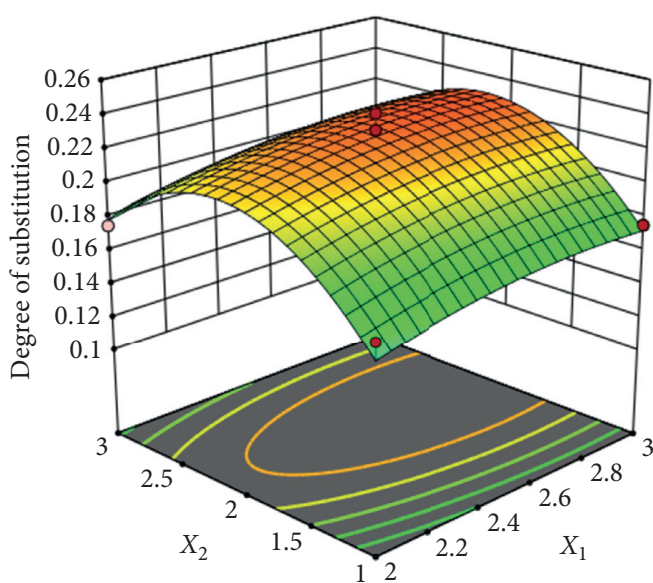

(a)

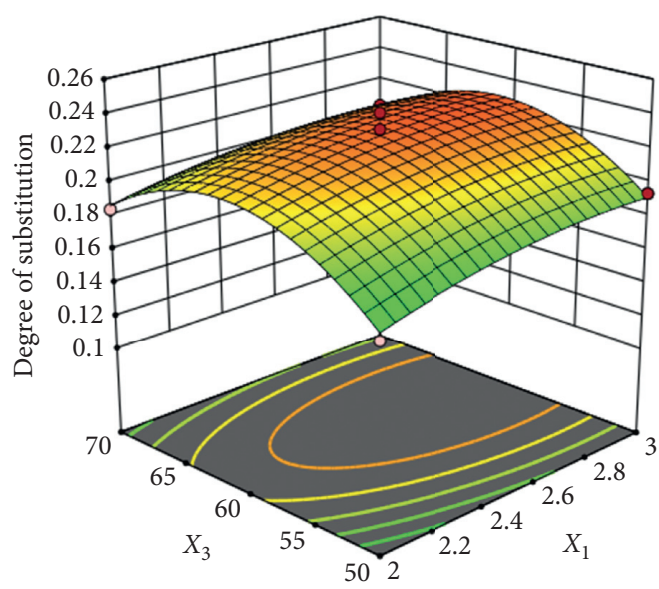

(c)

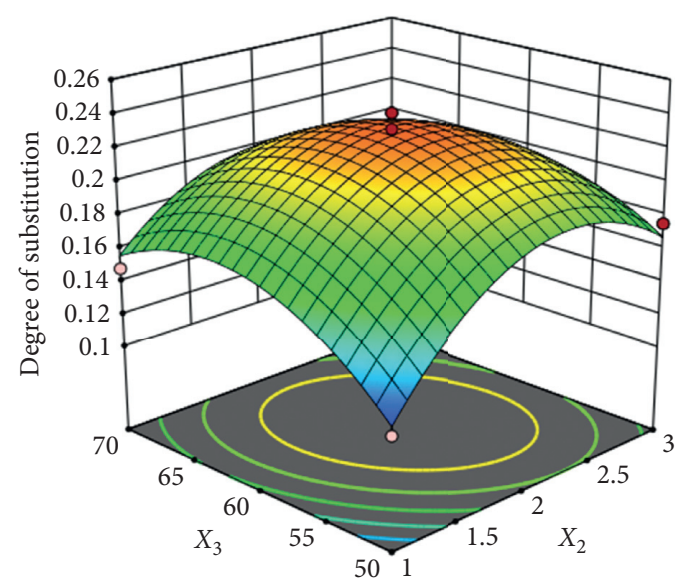

(e)

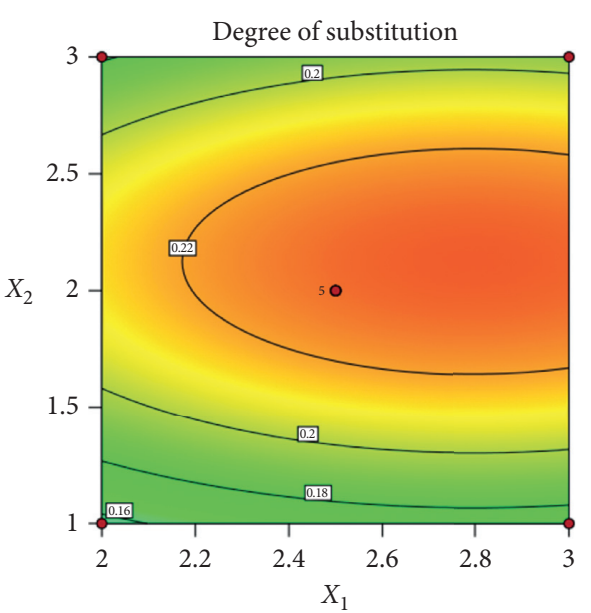

(b)

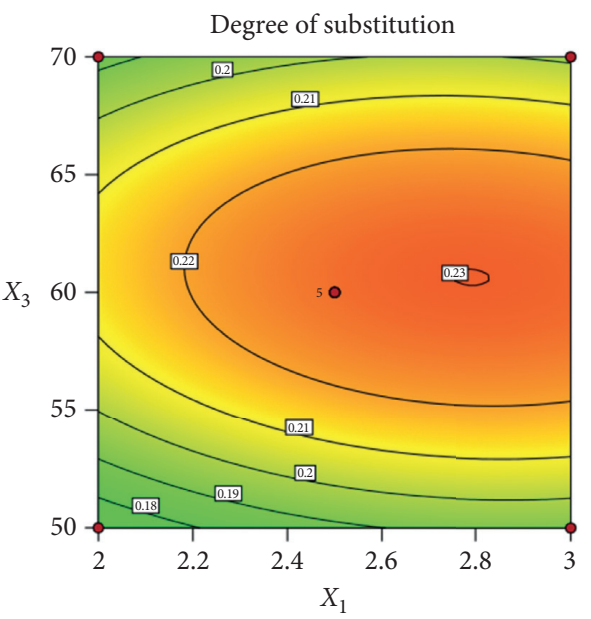

(d)

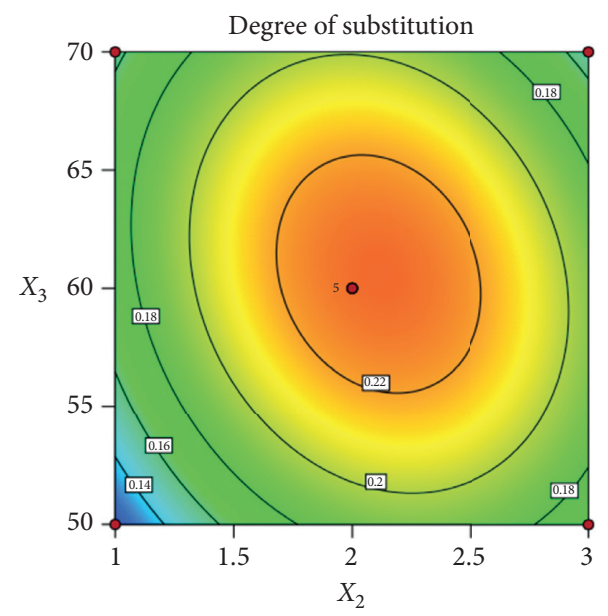

(f)

Figure 2: Response surface plots (a, c, e) and contour plots $(b, \mathrm{~d}, \mathrm{f})$ showing the effect of $\mathrm{NaOH}$ concentration (mol/L, $\left.X_{1}\right)$, chloroacetic acid content $\left(\%, X_{2}\right)$, and reaction temperature $\left({ }^{\circ} \mathrm{C}, X_{3}\right)$ on DS of CMJP.

that the mutual interactions between ratio of reaction temperature and chloroacetic acid content were significant.

According to software analysis, the optimal preparation conditions for CMJP were $\mathrm{NaOH}$ solution concentration of
$2.79 \mathrm{~mol} / \mathrm{L}$, chloroacetic acid content of $2.12 \%$, reaction temperature of $60.4^{\circ} \mathrm{C}$, and DS of 0.2307 . Three verification experiments were carried out in order to validate the adequacy of the model equation. Taking account of the 
operating convenience, the optimal preparation parameters of CMJP were determined as follows: the concentration of $\mathrm{NaOH}$ solution was $2.8 \mathrm{~mol} / \mathrm{L}$, the content of chloroacetic acid was $2.12 \%$, and the reaction temperature was $60.4^{\circ} \mathrm{C}$. By these parameters, the DS of CMJP was $0.2275 \pm 0.0108$, which is close to the maximum predicted value, indicating an excellent fit with the mathematical model.

\subsection{Characterization of Polysaccharides}

3.2.1. Rheological Behavior of JP and CMJP. The rheological behavior in terms of dynamic viscosity and shear rate of JP and CMJP is shown in Figure 3. The shear stress-shear rate relationships showed nonlinear; flow curves of JP and CMJP demonstrate a non-Newtonian behavior because polysaccharides form an intertwined network structure in the aqueous phase [27]. With the increase in shear rate, apparent viscosity of JP and CMJP decreased continuously and showed a characteristic of pseudoplastic fluids. However, shear stress increased with the rise of shear rate. Compared with JP, it was found that carboxymethylation reduced apparent viscosity and shear stress of JP.

3.2.2. Composition of JP and CMJP. After carboxymethylation, water solubility improved in CMJP. The content of total carbohydrate in JP $(75.33 \pm 0.11 \%)$ was relatively higher than that in CMJP $(69.22 \pm 0.32 \%)$, indicating that JP may have been hydrolyzed during the carboxymethylation process (Table 2 ).

As in previous studies, the Jinsixiaozao polysaccharides were shown to be largely composed of rhamnose (Rha), arabinose (Ara), xylose (Xyl), mannose (Man), glucose (Glc), and galactose (Gal). However, the molar ratio was different, which may be related to different raw materials, purification processes, and detection methods [7]. In our study, both JP and CMJP were heteropolysaccharides. JP was mainly composed of Rha, Ara, Xyl, Man, Glc, and Gal in the molar ratios of $0.31: 7.69: 0.54: 0.15: 1.08: 1$ and CMJP was mainly composed of Rha, Ara, Xyl, Glc, and Gal, with a molar ratio of $0.18,9.09,0.45,0.36$, and 1 , respectively. After carboxymethylation, the monosaccharide types have no significant changes expect Man (not detected in CMJP). Arabinose was also the major monosaccharide, but the CMJP had higher ratios of arabinose. Arabinose also was the main component monosaccharide in other jujube cultivars like "Shaanbeitanzao" [28], "Huanghetanzao" [13], and "Muzao" [14]. CMJP with high content of arabinose have good potential as antitumor [14], antioxidant, immunomodulation, and hepatoprotective agent [28]. For other monosaccharides, such as rhamnose, xylose, glucose, and galactose, the ratios were slightly decreased after modification. The result revealed that the carboxymethyl modification affects the physicochemical characteristics of JP but still retained the main component with almost the same monosaccharide types. The similar observations have been reported during the carboxymethylation of Cyclocarya paliurus polysaccharides [19].
3.2.3. Molecular Weight (Mw) Analysis. The weight-average molecular mass increased $10.62 \%$ from $2.75 \times 10^{5} \mathrm{Da}$ (JP) to $3.04 \times 10^{5} \mathrm{Da}(\mathrm{CMJP})$ after carboxymethylation. The raise might be owing to the incorporation of carboxymethylcontaining groups into the polymer structure during the carboxymethylation process. Wang et al. [29] also reported that the $M w$ of carboxymethylated polysaccharides extracted from Poria cocos sclerotium derivatives was higher than that of the natural polysaccharides.

3.2.4. FT-IR Spectrum Analysis. Figure 4 showed the FT-IR spectrum of JP and CMJP. In the range of $4000-400 \mathrm{~cm}^{-1}$, the FT-IR spectra of JP and CMJP were similar. The strong and wide peak near $3420 \mathrm{~cm}^{-1}$ was caused by $-\mathrm{OH}$ stretching; the adsorption bands of $2927 \mathrm{~cm}^{-1}$ and $2370 \mathrm{~cm}^{-1}$ were related to the stretching angular vibration of $\mathrm{C}-\mathrm{H}$. These peaks can all be corresponding to the characteristic absorption peaks of polysaccharides [30]. Three new strong absorption peaks were observed near $1600 \mathrm{~cm}^{-1}$, $1420 \mathrm{~cm}^{-1}$, and $1328 \mathrm{~cm}^{-1}$ which were attributed to the symmetric scale absorption of -COO after carboxymethyl modification [31]. The new peak at $1600 \mathrm{~cm}^{-1}$ was attributed to the angular vibration of $-\mathrm{COOH}$, while the absorption at wave number $1420 \mathrm{~cm}^{-1}$ corresponded to a vibrational absorption peak of $\mathrm{C}-\mathrm{H}$ on the methyl linked to the carboxyl group. The stretching vibrational absorption of carbonyl at $1328 \mathrm{~cm}-1$ was significantly enhanced, which indicated that the introduction of carboxymethyl $\left(-\mathrm{OCH}_{2}-\mathrm{COOH}\right)$ is successful. That means the natural polysaccharide was carboxymethylated successfully without change of the molecular structure of JP. The fingerprint region between 800 and $1200 \mathrm{~cm}^{-1}$ is known in carbohydrate. The spectrum shows bands at $890 \mathrm{~cm}^{-1}$ may be assigned to the $\mathrm{C}_{1}-\mathrm{H}$ deformation vibration of $\beta$-mannuronic acid residues. The band at $1012 \mathrm{~cm}^{-1}$ seems to be characteristic of uronic acid residues and bands at $1093 \mathrm{~cm}^{-1}$ may be assigned to the $\mathrm{C}-\mathrm{O}$ stretching vibrations of pyranose rings [32, 33].

3.2.5. UV-Vis Spectra. The UV scanning analysis of crude jujube polysaccharide, JP, and CMJP was shown in Figure 5. Strong absorption peaks at $260 \mathrm{~nm}-280 \mathrm{~nm}$ of crude jujube polysaccharides indicated the presence of nucleic acids and protein [30]. No absorption peak was detected at $260 \mathrm{~nm}-280 \mathrm{~nm}$, which means impurity was completely removed from both of JP and CMJP. There were no obvious differences between JP and CMJP from the spectrum.

3.2.6. SEM and EDS Analyses. Figures 6(a)-6(d) illustrated the scanning electron micrographs (SEM) of JP and CMJP at 5000x and 10,000x magnification. The morphology of CMJP showed significant variations in morphological properties compared with JP. JP occurred in a state of fibrous, layered, thin-sliced small particles, with a smooth surface. Single molecules and molecular groups linked together and formed polymers with a smooth fragmented appearance with various sizes. However, CMJP had a rough and uneven spherical shape with netted and loose porous structures, 


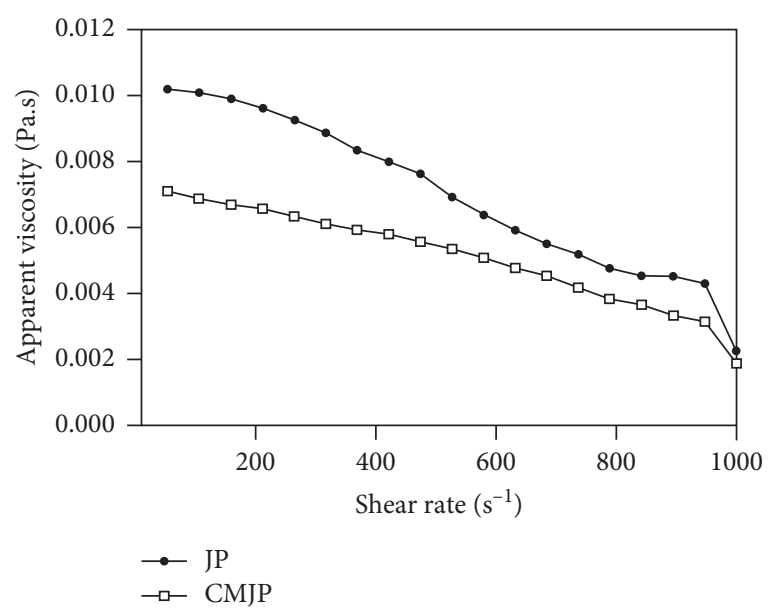

(a)

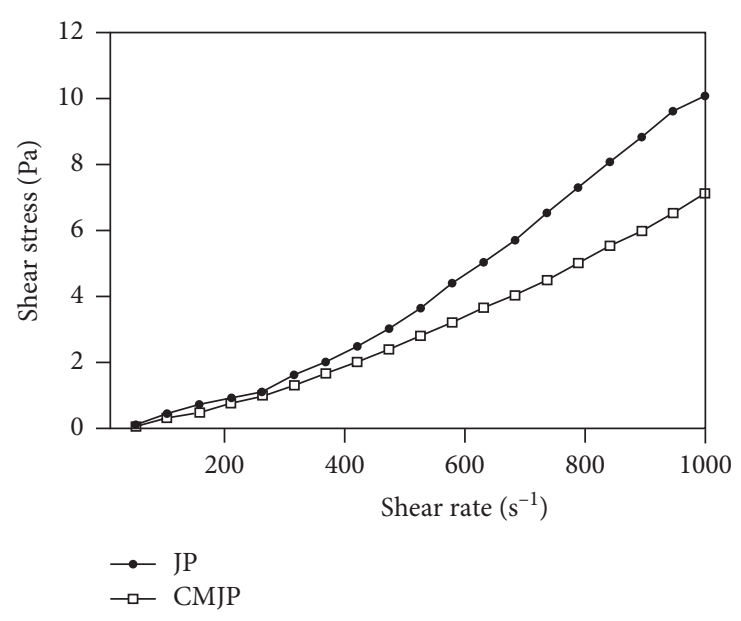

(b)

FIGURE 3: Effect of carboxymethylation modification on apparent viscosity (a) and shear stress (b) of JP and CMJP aqueous solution at $25^{\circ} \mathrm{C}$.

TABLE 2: Water solubility, viscosity, and the chemical composition of polysaccharide from JP and CMJP.

\begin{tabular}{|c|c|c|c|c|c|c|c|c|c|}
\hline \multirow{2}{*}{ Sample } & \multirow{2}{*}{$\begin{array}{l}\text { Water solubility (mg/ } \\
\mathrm{mL})\end{array}$} & \multirow{2}{*}{$\begin{array}{l}\text { Total carbohydrate } \\
(\%)\end{array}$} & \multirow{2}{*}{$\begin{array}{l}\text { Uronic acid } \\
\quad(\%)\end{array}$} & \multicolumn{6}{|c|}{ Monosaccharide composition (\%) } \\
\hline & & & & Rhamnose & Arabinose & Xylose & Mannose & Glucose & Galactose \\
\hline JP & $50.8 \pm 0.93$ & $75.33 \pm 0.11$ & $39.35 \pm 0.10$ & 0.31 & 7.69 & 0.54 & 0.15 & 1.08 & 1 \\
\hline CMJP & $85.1 \pm 1.27$ & $69.22 \pm 0.32$ & $40.38 \pm 0.15$ & 0.18 & 9.09 & 0.45 & ND & 0.36 & 0.98 \\
\hline
\end{tabular}

Note. ND: not detected.

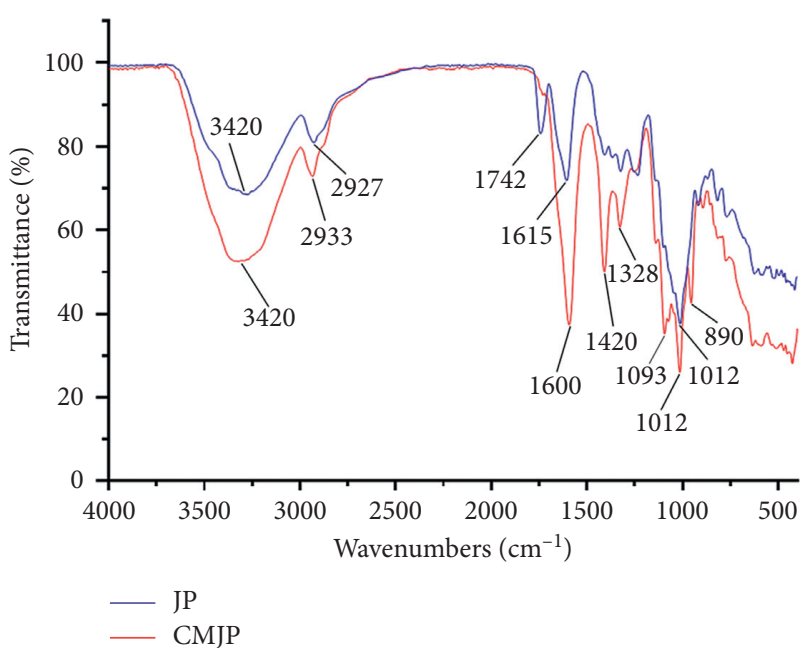

FIGURE 4: Fourier transform infrared spectra (FT-IR) of JP and CMJP.

which indicated there was no close binding, with repulsive forces between molecules and reduced intermolecular attraction. Li et al. [34] reported that the SEM of carboxymethylated corn bran polysaccharide exhibited a relatively rough appearance with some pores and featured large wrinkles on the surface, which was in accordance with our results.

The designated points in JP (Figure 6(e)) and CMJP (Figure 6(f)) were analyzed by EDS to further determine the elemental composition after carboxymethylation. JP mainly contain $\mathrm{C}$ and $\mathrm{O}$ elements while CMJP mainly contain $\mathrm{C}, \mathrm{O}$, and $\mathrm{Na}$ elements. The presence of $\mathrm{Na}$ in $\mathrm{CMJP}$ was due to the use of $\mathrm{NaOH}$ in carboxymethylation. Increasing of $\mathrm{O} / \mathrm{C}$ ratio in CMJP was attributed to the introduction of $-\mathrm{COOH}$.

3.3. In Vitro Antioxidant Activities of Polysaccharide. $\mathrm{DPPH}$ is a stable free radical which could be reduced on acceptance of hydrogen donated by the antioxidant and converted to DPPH-H (the nonradical from of DPPH) [35]. As shown in Figure $7(\mathrm{a})$, in the range of $0.1-5 \mathrm{mg} / \mathrm{mL}$, the scavenging activities of the two polysaccharides were lower than vitamin $\mathrm{C}\left(\mathrm{IC}_{50}=0.28 \mathrm{mg} / \mathrm{mL}\right)$. The $\mathrm{IC}_{50}$ value of CMJP $(1.87 \mathrm{mg} / \mathrm{mL})$ for eliminating $\mathrm{DPPH}$ radicals was higher than JP $(1.59 \mathrm{mg} / \mathrm{mL})$. The carboxymethylated derivatives of Ganoderma atrum polysaccharide are weaker in scavenging DPPH free radicals than natural derivatives [36]. At the concentration of $5 \mathrm{mg} / \mathrm{mL}$, JP $(90.47 \pm 0.04 \%)$ showed higher scavenging activity than CMJP (86.02 $\pm 0.85 \%)$. The scavenging capacity of DPPH radical was mainly ascribed to the electron donation power to the free radicals [37]. Carboxymethylation modification did not enhance the scavenging ability of DPPH radicals of polysaccharides; the same results were obtained on the carboxymethylation modification of Ganoderma atrum polysaccharide [36] and Phellinus linteus polysaccharide [38].

Hydroxyl radical is well known as a strong oxidant which can react with most of biomolecules in living cells and resulting in severe damage or cell death [35]. As shown in Figure $7(\mathrm{~b})$, in the range of $1-5 \mathrm{mg} / \mathrm{mL}$, the two polysaccharides have been found to possess the hydroxyl radical scavenging activity in a concentration-dependent manner 


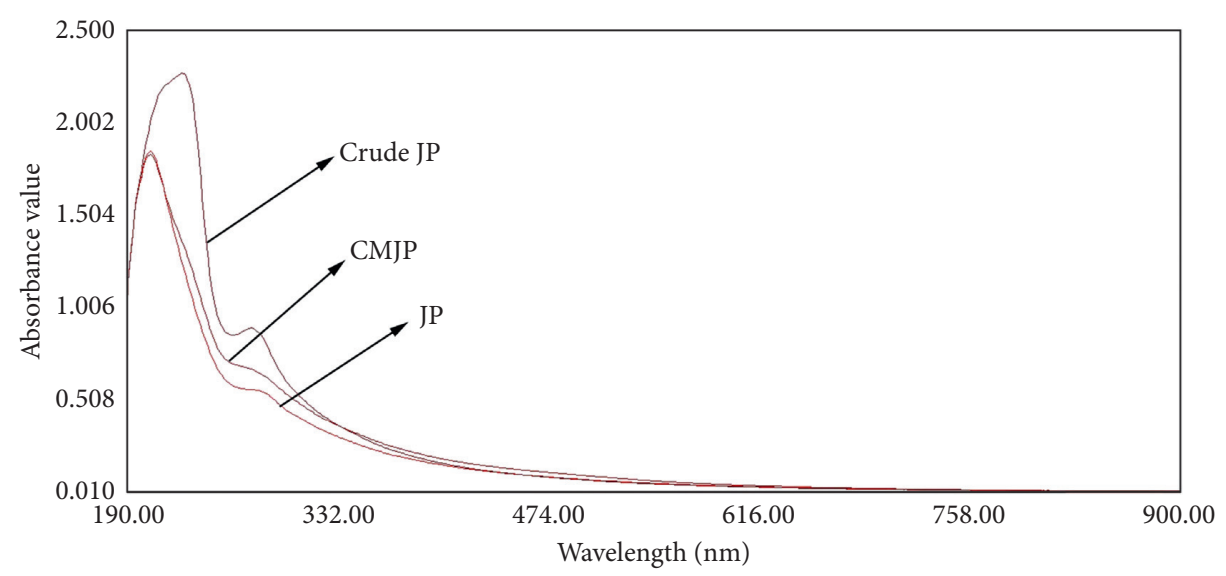

FIGURE 5: Ultraviolet and visible spectrophotometry (UV-Vis) spectra of crude JP, JP, and CMJP were recorded in the range of 190-900 nm.

lower than positive control (vitamin $\mathrm{C}, \mathrm{IC}_{50}=0.32 \mathrm{mg} / \mathrm{mL}$ ). CMJP $\quad\left(\mathrm{IC}_{50}=1.64 \mathrm{mg} / \mathrm{mL}\right)$ showed significantly higher scavenging effect compared to JP $\left(\mathrm{IC}_{50}=2.46 \mathrm{mg} / \mathrm{mL}\right.$, $P<0.01$ ). These results indicated that the insertion of carboxymethyl has a noticeable effect on the scavenging abilities of hydroxyl radical. The scavenging rates of JP and CMJP were $37.34 \pm 0.68 \%$ and $79.44 \pm 1.06 \%$ while that of vitamin $\mathrm{C}$ was $99.24 \pm 0.14 \%$ at the concentration of $5 \mathrm{mg} / \mathrm{mL}$. CMJP has a strong hydroxyl radical scavenging ability and reached $80.05 \%$ of vitamin C, which indicated that CMJP could be explored as a potential antioxidant. The hydroxyl radical scavenging activity could be influenced by the donor of electron or hydrogen [39]. Xu et al. [8] reported that carboxymethylation modification significantly enhanced the hydroxyl radical scavenging ability of Ganoderma lucidum polysaccharides $(83.7 \%$ at $5 \mathrm{mg} / \mathrm{mL})$, which is in accordance with our results.

Superoxide radical is considered as an initial free radical, easily transformed from molecular oxygen and causes other cell damaging free radicals $\left(\mathrm{H}_{2} \mathrm{O}_{2}\right.$, hydroxyl radicals) in living systems [40]. As shown in Figure 7(c), with an increase in concentration $(1-5 \mathrm{mg} / \mathrm{mL})$, the scavenging abilities of JP and CMJP were lower than that of vitamin $\mathrm{C}$ and that of CMJP was the weakest. The $\mathrm{IC}_{50}$ value of CMJP $(2.59 \mathrm{mg} / \mathrm{mL})$ for reducing superoxide radicals was higher than JP $(1.77 \mathrm{mg} / \mathrm{mL})$. According to reports, Cyclocarya paliurus polysaccharide also has a low scavenging effect on superoxide free radicals after carboxymethylation modification [19], which is consistent with the results of this study.

The electron-donating ability of antioxidants was measured using the potassium ferricyanide reduction method. As shown in Figure $7(\mathrm{~d})$, in the range of $1-5 \mathrm{mg} / \mathrm{mL}$, JP $\left(\mathrm{IC}_{50}=2.12 \mathrm{mg} / \mathrm{mL}\right)$ and $\mathrm{CMJP}\left(\mathrm{IC}_{50}=2.60 \mathrm{mg} / \mathrm{mL}\right)$ showed a poor reducing ability compared with vitamin $\mathrm{C}$ $\left(\mathrm{IC}_{50}=0.18 \mathrm{mg} / \mathrm{mL}\right)$. The reduction ability of JP was only $1.20 \pm 0.05 \%$ at the concentration of $5 \mathrm{mg} / \mathrm{mL}$, and the reduction ability of CMJP was significantly lower than JP $(0.94 \pm 0.01 \%)$. The reduction ability of JP could not be improved by carboxymethylation modification, and the same results were reported in cucumber polysaccharide [41].
In the present study, carboxymethylated modification could reduce DPPH radicals, superoxide radicals, and power but increase hydroxyl radical scavenging activity of Jinsixiaozao polysaccharides. Previous studies have suggested that the antioxidant activity may be related to monosaccharide composition, $\mathrm{Mw}$, and structure of different kinds of polysaccharides [39, 42]. It was not a function of a single factor but a combination of several factors that might be responsible for the changes of antioxidant activities. The accurate clarification of mechanism underlying the antioxidant activity by JP and CMJP is still not fully understood, and the further in-depth works are in progress by our team.

3.4. Prebiotic Activities of JP and CMJP. Grow curves at $\mathrm{pH}$ $5.7 \pm 0.2$ of the three Lactobacillus strains were shown in Figure 8. The three Lactobacillus strains reached exponential growth phase after $4 \mathrm{~h}$ incubation. L. acidophilus, L. plantarum, and L. rhamnosus transitioned to stationary phase after $16 \mathrm{~h}, 16 \mathrm{~h}$, and $18 \mathrm{~h}$ incubation, respectively. All of the three Lactobacillus strains reached stationary phase after $24 \mathrm{~h}$ incubation which were in accordance with the previous studies [43-45]. The effects of JP and CMJP on the proliferation of $L$. acidophilus, $L$. plantarum, and L. rhamnosus after $6 \mathrm{~h}, 10 \mathrm{~h}, 16 \mathrm{~h}$, and $24 \mathrm{~h}$ incubation were shown in Figure 9. The populations of Lactobacillus strains raised with incubation time. Both of JP and CMJP were found to have a significant effect on the growth of probiotics, compared to control at all concentrations tested. For all tested times, it was observed that the growth of all tested Lactobacillus strains was remarkably higher on CMJP than $\mathrm{JP}$, especially for $L$. plantarum. In addition, after $24 \mathrm{~h}$ incubation with the presence of increased concentration of CMJP as a carbon source, L. plantarum and L. rhamnosus showed an outstanding higher growth rate compared with CMJP that significantly increased the prebiotic activities of JP for all the Lactobacillus strains.

Wang et al. [46] reported that rapeseed polysaccharide with higher sugar content and lower $M w$ exert better proliferative effect on probiotic growth. However, our 


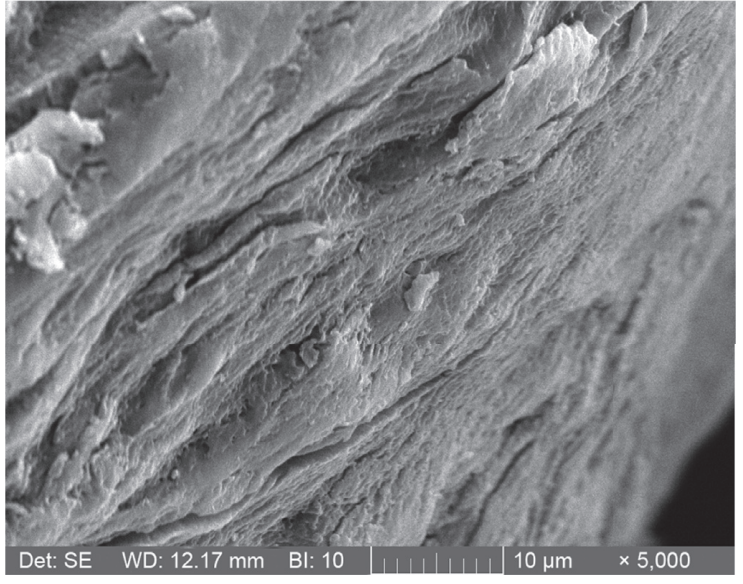

(a)

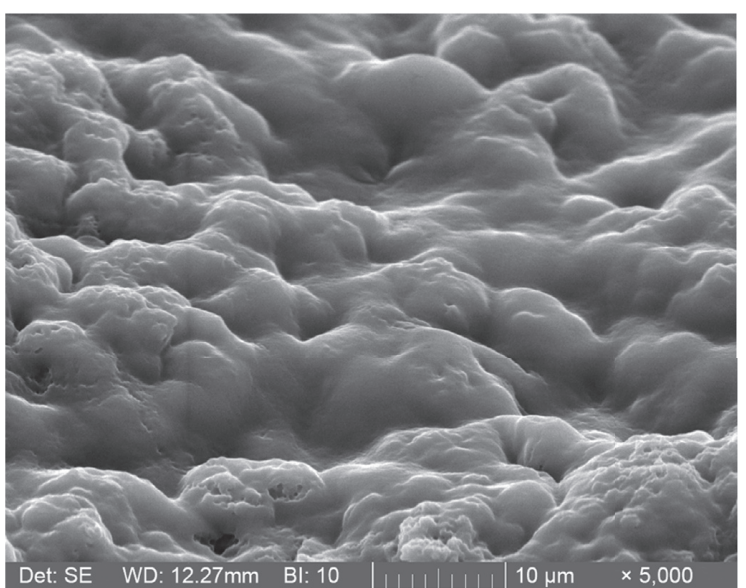

(c)

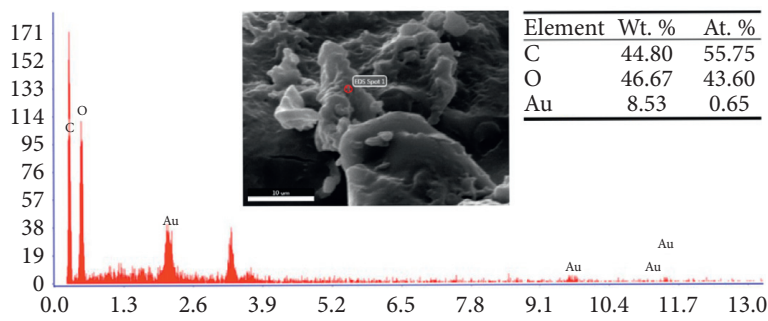

(e)

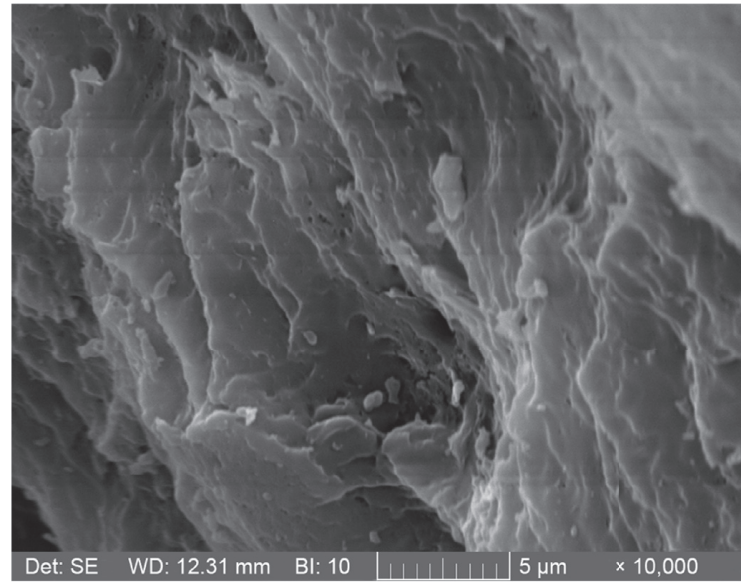

(b)

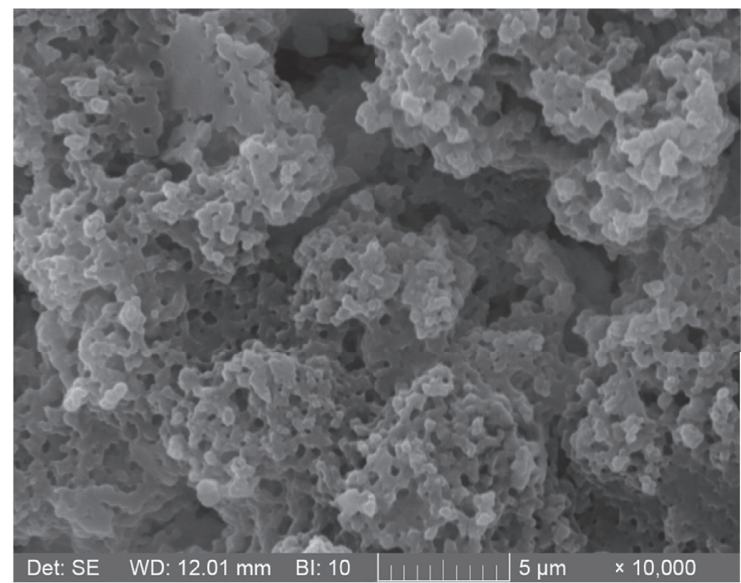

(d)

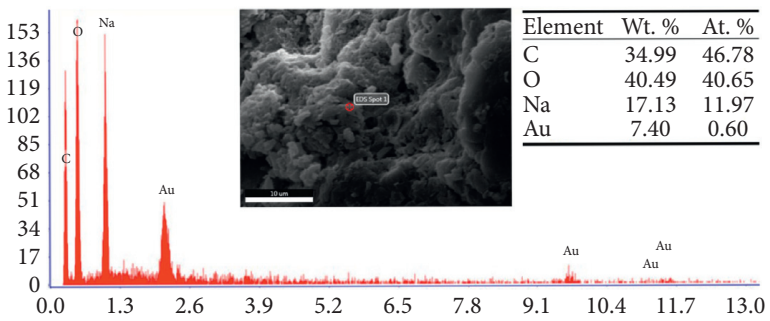

(f)

FIGURE 6: Scanning electron micrographs (SEM) and energy-dispersive X-ray spectroscopy (EDS) analysis of polysaccharide. (a) 5000x; (b) 10000x; (e) JP-point and carboxymethylated polysaccharide (c) 5000x; (d) 10000x; (f) CMJP-point from Ziziphus Jujuba cv. Jinsixiaozao.

analysis suggested that JP with higher sugar content and lower $M w$ showed weaker proliferative effect compared with CMJP. This could be due to the different physicochemical properties of different kinds of polysaccharides which need to be further investigated. Previous work also reported that more branch degree, good water solubility, and lower viscosity polysaccharides could more easily, rapidly, and completely be utilized by probiotics [46, 47]. CMJP had better prebiotic activity due to its significantly improved water solubility after the addition of carboxymethyl groups. In addition, previous studies have also shown that carbohydrates composed of arabinose, glucose, galactose, and xylose generally showed better prebiotic activities $[47,48]$. JP and CMJP composed of the abovementioned monosaccharides, so both of them could promote the growth of probiotics. Besides, the different molar ratio of the monosaccharides in JP and CMJP might also attribute to their different prebiotic effects [46].

In this study, both of JP and CMJP possessed the potential as prebiotics and CMJP showed stronger prebiotic activities than JP. The mechanisms need to be disclosed, and future study will be continued to explore the in vivo prebiotic activities of JP and CMJP using animal model. 

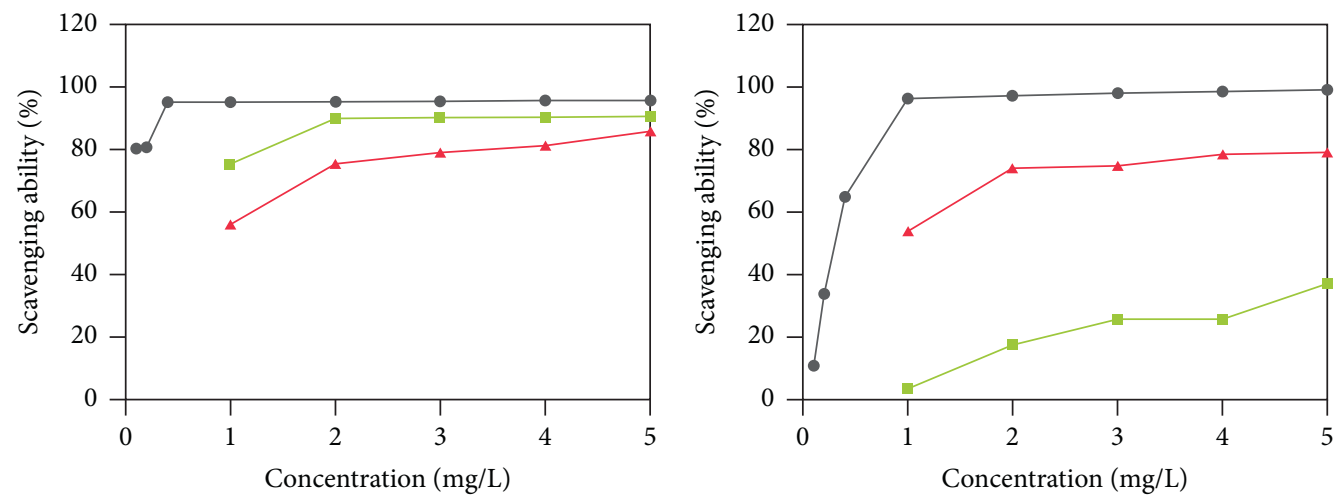

$\rightarrow$ Vitamin C

$\because \mathrm{JP}$

$\rightarrow$ Vitamin C

- JP

$\multimap$ CMJP

(a)

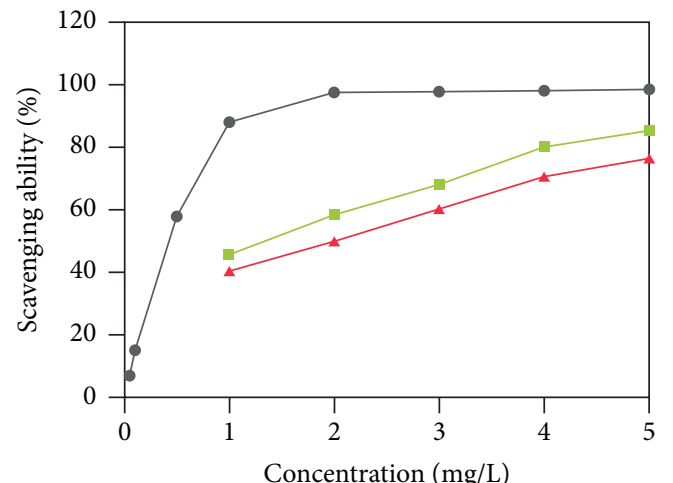

(b)

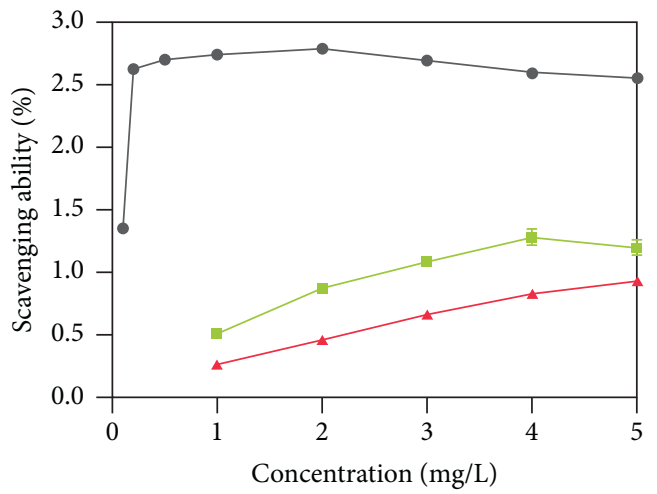

$\rightarrow$ Vitamin C

$\rightarrow$ JP

$\neg$ CMJP

$\rightarrow$ Vitamin C

$\because \mathrm{JP}$

$\leftarrow$ CMJP

(c)

(d)

FIGURE 7: Scavenging effect of JP and CMJP on DPPH radicals (a), hydroxyl radicals (b), superoxide radicals (c), and reducing power (d) compared with that of vitamin C. Results were expressed as means \pm SD of three parallel measurements.

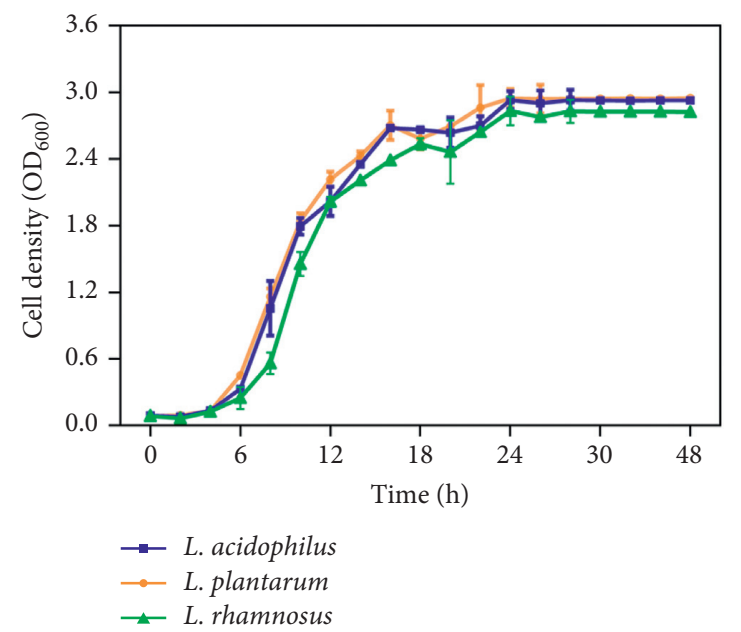

FIgURe 8: Growth curves of L. acidophilus, L. plantarum, and L. rhamnosus in MRS medium at $37^{\circ} \mathrm{C}(\mathrm{pH}=5.7 \pm 0.2)$. 


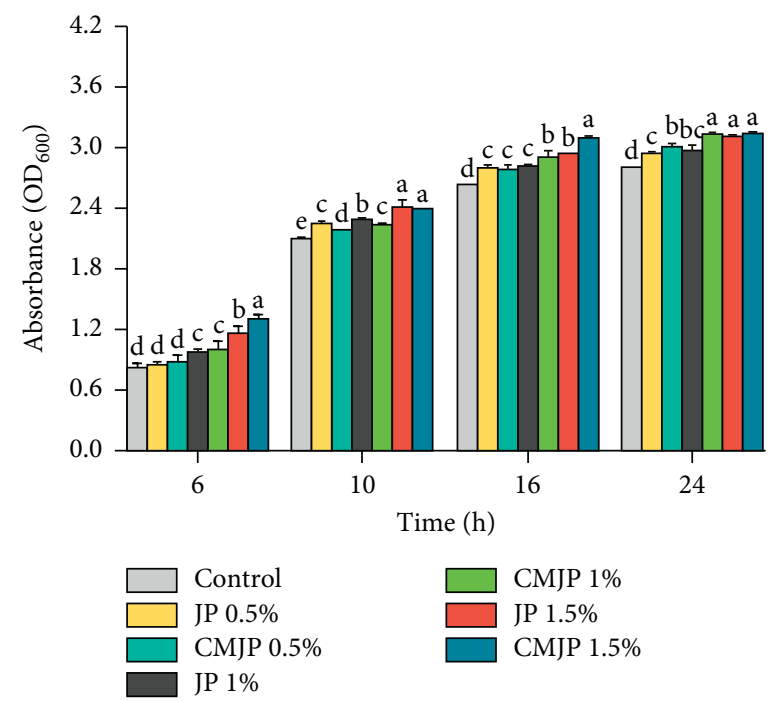

(a)

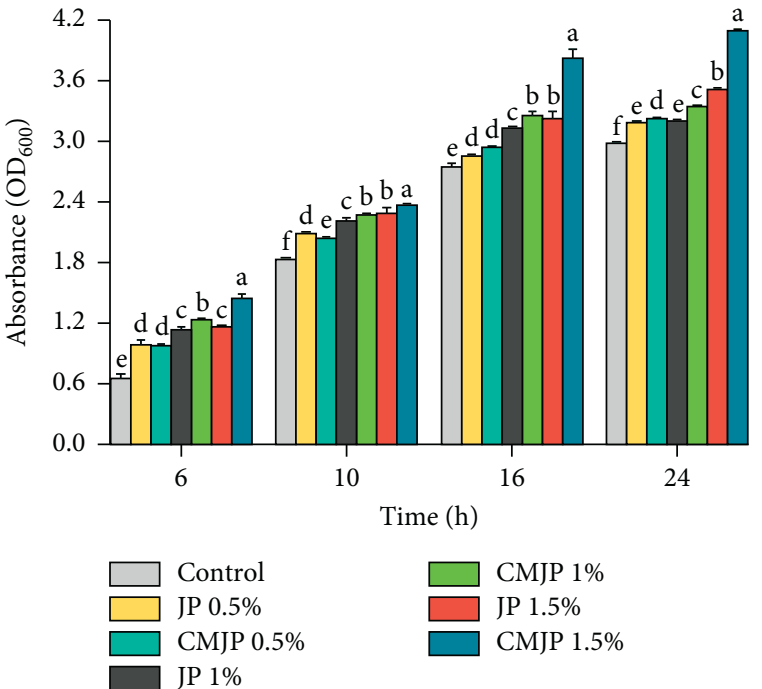

(b)

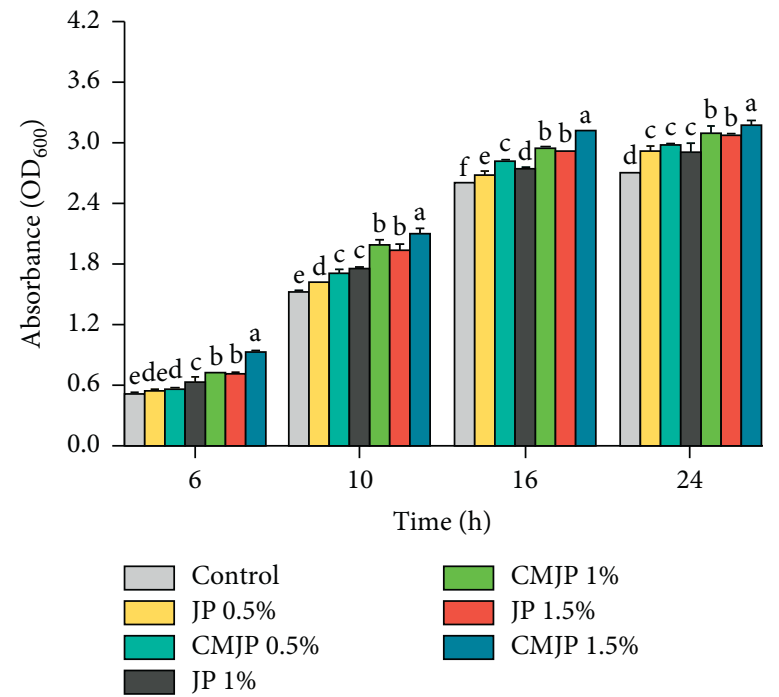

(c)

Figure 9: Effects of JP and CMJP on the proliferation of Lactobacillus strains after $6 \mathrm{~h}, 10 \mathrm{~h}, 16 \mathrm{~h}$, and $24 \mathrm{~h}$ incubation. L. acidophilus (a), L. plantarum (b), and L. rhamnosus (c). Data with different lowercase letters represent significant differences at $P<0.05$.

\section{Conclusions}

In this study, carboxymethylated Jinsixiaozao polysaccharides with high degree of substitution were successfully prepared. Compared with JP, introduction of carboxymethyl groups not only affected the physicochemical and structural characteristics, but promoted in vitro antioxidant and prebiotic activities. CMJP is a heteropolysaccharide, mainly composed of galacturonic acid, arabinose, and a small amount of rhamnose, xylose, glucose, and galactose. It exhibits higher water solubility, lower total carbohydrate content, and higher Mw. Both JP and CMJP are pseudoplastic fluids, and carboxymethylation modification can reduce the apparent viscosity and shear stress of jujube polysaccharides. FT-IR spectra showed that the carboxymethylation modification was successful, and the carboxymethylation modification can significantly enhance the hydroxyl radical scavenging activity of Jinsixiaozao polysaccharides. In addition, carboxymethylation can improve the proliferation of JP on L. acidophilus, L. plantarum, and L. rhamnosus. These results indicate that carboxymethylated jujube polysaccharide can be developed and utilized as a potential antioxidant and prebiotic. CMJP could be exploited as novel functional ingredient which is natural and safe. However, its application in functional foods or medicines needs to be combined with animal models to further study its safety in vivo.

\section{Data Availability}

The data used to support the study are included within the article. Raw data can be acquired from the corresponding author upon reasonable request (aocw@163.com and lyzhihuizhao@126.com). 


\section{Disclosure}

Jingjing Kou is the co-first author.

\section{Conflicts of Interest}

The authors declare that there are no conflicts of interest regarding the publication of this paper.

\section{Authors' Contributions}

Runfang Feng and Jingjing Kou contributed equally to this work.

\section{Acknowledgments}

This research was financially supported by the Natural Science Foundation of Hebei Province (C2019204366) and the Key R\&D Projects in Hebei Province (20327123D), Top Young Talents of Hebei Province.

\section{Supplementary Materials}

Table S1: Box-Behnken experimental design and the results for degree of substitution (DS) of CMJP polysaccharides $(n=3)$. (Supplementary Materials)

\section{References}

[1] J. Liu, S. Willför, and C. Xu, "A review of bioactive plant polysaccharides: biological activities, functionalization, and biomedical applications," Bioactive Carbohydrates and Dietary Fibre, vol. 5, no. 1, pp. 31-61, 2015.

[2] A. Zong, H. Cao, and F. Wang, "Anticancer polysaccharides from natural resources: a review of recent research," Carbohydrate Polymers, vol. 90, no. 4, pp. 1395-1410, 2012.

[3] Y. Cai, P. Chen, C. Wu, J. Duan, and H. Bai, "Sulfated modification and biological activities of polysaccharides derived from Zizyphus jujuba cv. Jinchangzao," International Journal of Biological Macromolecules, vol. 120, pp. 1149-1155, 2018.

[4] Y.-T. Li, B.-J. Chen, W.-D. Wu et al., "Antioxidant and antimicrobial evaluation of carboxymethylated and hydroxamated degraded polysaccharides from Sargassum fusiforme," International Journal of Biological Macromolecules, vol. 118, pp. 1550-1557, 2018.

[5] Y. Deng, M. Li, L.-X. Chen et al., "Chemical characterization and immunomodulatory activity of acetylated polysaccharides from Dendrobium devonianum," Carbohydrate Polymers, vol. 180, pp. 238-245, 2018.

[6] Z. Wang, J. Xie, M. Shen, S. Nie, and M. Xie, "Sulfated modification of polysaccharides: synthesis, characterization and bioactivities," Trends in Food Science \& Technology, vol. 74, pp. 147-157, 2018.

[7] X. Ji, Q. Peng, Y. Yuan, J. Shen, X. Xie, and M. Wang, "Isolation, structures and bioactivities of the polysaccharides from jujube fruit (Ziziphus jujuba Mill.): a review," Food Chemistry, vol. 227, pp. 349-357, 2017.

[8] J. Xu, W. Liu, W. Yao, X. Pang, D. Yin, and X. Gao, "Carboxymethylation of a polysaccharide extracted from Ganoderma lucidum enhances its antioxidant activities in vitro," Carbohydrate Polymers, vol. 78, no. 2, pp. 227-234, 2009.
[9] W. Zhu, S. Zhou, J. Liu, R. J. C. McLean, and W. Chu, "Prebiotic, immuno-stimulating and gut microbiota-modulating effects of Lycium barbarum polysaccharide," Biomedicine \& Pharmacotherapy, vol. 121, Article ID 109591, 2020.

[10] Q.-H. Gao, C.-S. Wu, and M. Wang, “The jujube (Ziziphus jujuba Mill.) fruit: a review of current knowledge of fruit composition and health benefits," Journal of Agricultural and Food Chemistry, vol. 61, no. 14, pp. 3351-3363, 2013.

[11] H. Rostami and S. M. T. Gharibzahedi, "Microwave-assisted extraction of jujube polysaccharide: optimization, purification and functional characterization," Carbohydrate Polymers, vol. 143, pp. 100-107, 2016.

[12] G. Cui, W. Zhang, Q. Wang et al., "Extraction optimization, characterization and immunity activity of polysaccharides from Fructus Jujubae," Carbohydrate Polymers, vol. 111, pp. 245-255, 2014

[13] G. Liu, X. Liu, Y. Zhang et al., "Hepatoprotective effects of polysaccharides extracted from Zizyphus jujube cv. Huanghetanzao," International Journal of Biological Macromolecules, vol. 76, pp. 169-175, 2015.

[14] Y. Wang, X. Liu, J. Zhang et al., "Structural characterization and in vitro antitumor activity of polysaccharides from Zizyphus jujuba cv. Muzao," RSC Advances, vol. 5, no. 11, pp. 7860-7867, 2015.

[15] Z. Zhao, J. Li, X. Wu et al., "Structures and immunological activities of two pectic polysaccharides from the fruits of Ziziphus jujuba Mill. cv. Jinsixiaozao Hort," Food Research International, vol. 39, no. 8, pp. 917-923, 2006.

[16] J. Li, Y. Liu, L. Fan, L. Ai, and L. Shan, "Antioxidant activities of polysaccharides from the fruiting bodies of Zizyphus Jujuba cv. Jinsixiaozao," Carbohydrate Polymers, vol. 84, no. 1, pp. 390-394, 2011.

[17] J. Li, L. Shan, Y. Liu, L. Fan, and L. Ai, "Screening of a functional polysaccharide from Zizyphus Jujuba cv. Jinsixiaozao and its property," International Journal of Biological Macromolecules, vol. 49, no. 3, pp. 255-259, 2011.

[18] A. Staub, "Removeal of protein-Sevag method," Methods Carbohydrate Chemistry, vol. 5, pp. 5-6, 1965.

[19] Z.-J. Wang, J.-H. Xie, M.-Y. Shen et al., "Carboxymethylation of polysaccharide from Cyclocarya paliurus and their characterization and antioxidant properties evaluation," Carbohydrate Polymers, vol. 136, pp. 988-994, 2016.

[20] Y.-Y. Cao, Y.-H. Ji, A.-M. Liao et al., "Effects of sulfated, phosphorylated and carboxymethylated modifications on the antioxidant activities in-vitro of polysaccharides sequentially extracted from Amana edulis," International Journal of Biological Macromolecules, vol. 10, Article ID 10410, 2020.

[21] Z. Duan, W. Duan, F. Li, Y. Li, P. Luo, and H. Liu, "Effect of carboxymethylation on properties of fucoidan from Laminaria japonica: antioxidant activity and preservative effect on strawberry during cold storage," Postharvest Biology and Technology, vol. 151, pp. 127-133, 2019.

[22] Y. Liu and G. Huang, "The antioxidant activities of carboxymethylated cushaw polysaccharide," International Journal of Biological Macromolecules, vol. 121, pp. 666-670, 2019.

[23] Z. Wu, P. Zhou, J. Yang, and J. Li, "Determination of the optimal reaction conditions for the preparation of highly substituted carboxymethyl Cassia tora gum," Carbohydrate Polymers, vol. 157, pp. 527-532, 2017.

[24] G. Joshi, S. Naithani, V. K. Varshney, S. S. Bisht, V. Rana, and P. K. Gupta, "Synthesis and characterization of carboxymethyl cellulose from office waste paper: a greener approach towards waste management," Waste Management, vol. 38, pp. 33-40, 2015. 
[25] W. Chen, W.-P. Wang, H.-S. Zhang, and Q. Huang, "Optimization of ultrasonic-assisted extraction of water-soluble polysaccharides from Boletus edulis mycelia using response surface methodology," Carbohydrate Polymers, vol. 87, no. 1, pp. 614-619, 2012.

[26] X. Guo, X. Zou, and M. Sun, "Optimization of extraction process by response surface methodology and preliminary characterization of polysaccharides from Phellinus igniarius," Carbohydrate Polymers, vol. 80, no. 2, pp. 344-349, 2010.

[27] F. Hentati, G. Pierre, A. V. Ursu et al., "Rheological investigations of water-soluble polysaccharides from the Tunisian brown seaweed Cystoseira compressa," Food Hydrocolloids, vol. 103, Article ID 105631, 2020.

[28] D. Wang, Y. Zhao, Y. Jiao, L. Yu, S. Yang, and X. Yang, "Antioxidative and hepatoprotective effects of the polysaccharides from Zizyphus jujube cv. Shaanbeitanzao," Carbohydrate Polymers, vol. 88, no. 4, pp. 1453-1459, 2012.

[29] Y. Wang, C. Zhong, J. Mao, M. Fan, and X. Wu, "Optimization of ultrasonic-assisted extraction process of Poria cocos polysaccharides by response surface methodology," Carbohydrate Polymers, vol. 77, pp. 713-717, 2009.

[30] X. Ji, F. Liu, Q. Peng, and M. Wang, "Purification, structural characterization, and hypolipidemic effects of a neutral polysaccharide from Ziziphus Jujuba cv. Muzao," Food Chemistry, vol. 245, pp. 1124-1130, 2018.

[31] L. Fan, L. Wang, S. Gao et al., "Synthesis, characterization and properties of carboxymethyl kappa carrageenan," Carbohydrate Polymers, vol. 86, no. 3, pp. 1167-1174, 2011.

[32] N. Chandía, B. Matsuhiro, and A. Vásquez, "Alginic acids in Lessonia trabeculata: characterization by formic acid hydrolysis and FT-IR spectroscopy," Carbohydrate Polymers, vol. 46, no. 1, pp. 81-87, 2001.

[33] N. P. Chandía, B. Matsuhiro, E. Mejías, and A. Moenne, "Alginic acids in Lessonia vadosa: partial hydrolysis and elicitor properties of the polymannuronic acid fraction," Journal of Applied Phycology, vol. 16, no. 2, pp. 127-133, 2004.

[34] J. Li, W. Shang, X. Si et al., "Carboxymethylation of corn bran polysaccharide and its bioactive property," International Journal of Food Science \& Technology, vol. 52, no. 5, pp. 1176-1184, 2017.

[35] Z.-Y. Zhao, Q. Zhang, Y.-F. Li, L.-L. Dong, and S.-L. Liu, "Optimization of ultrasound extraction of Alisma orientalis polysaccharides by response surface methodology and their antioxidant activities," Carbohydrate Polymers, vol. 119, pp. 101-109, 2015.

[36] Y. Chen, H. Zhang, Y. Wang, S. Nie, C. Li, and M. Xie, "Acetylation and carboxymethylation of the polysaccharide from Ganoderma atrum and their antioxidant and immunomodulating activities," Food Chemistry, vol. 156, pp. 279288, 2014.

[37] J.-H. Xie, M.-Y. Shen, M.-Y. Xie et al., "Ultrasonic-assisted extraction, antimicrobial and antioxidant activities of Cyclocarya paliurus (Batal.) Iljinskaja polysaccharides," Carbohydrate Polymers, vol. 89, no. 1, pp. 177-184, 2012.

[38] J.-Y. Shin, S. Lee, I. Y. Bae, S.-H. Yoo, and H. G. Lee, "Structural and biological study of Carboxymethylated Phellinus linteus Polysaccharides," Journal of Agricultural and Food Chemistry, vol. 55, no. 9, pp. 3368-3372, 2007.

[39] M.-J. Shi, X. Wei, J. Xu et al., "Carboxymethylated degraded polysaccharides from Enteromorpha prolifera: preparation and in vitro antioxidant activity," Food Chemistry, vol. 215, pp. 76-83, 2017.

[40] J. A. Vaz, L. Barros, A. Martins, C. Santos-Buelga, M. H. Vasconcelos, and I. C. F. R. Ferreira, "Chemical composition of wild edible mushrooms and antioxidant properties of their water soluble polysaccharidic and ethanolic fractions," Food Chemistry, vol. 126, no. 2, pp. 610-616, 2011.

[41] S. Chen, H. Huang, and G. Huang, "Extraction, derivatization and antioxidant activity of cucumber polysaccharide," International Journal of Biological Macromolecules, vol. 140, pp. 1047-1053, 2019.

[42] Y. Liu, G. Huang, and J. Hu, "Extraction, characterisation and antioxidant activity of polysaccharides from Chinese watermelon," International Journal of Biological Macromolecules, vol. 111, pp. 1304-1307, 2018.

[43] D. Olson and K. Aryana, "Effect of prebiotics on Lactobacillus acidophilus growth and resulting $\mathrm{pH}$ changes in skim milk and a model peptone system," Journal of Microbial \& Biochemical Technology, vol. 4, pp. 121-125, 2012.

[44] Z. Matejčeková, D. Liptáková, S. Spodniaková, and Ľ. Valík, "Characterization of the growth of Lactobacillus plantarum in milk in dependence on temperature," Acta Chimica Slovaca, vol. 9, pp. 104-108, 2016.

[45] L. Cai, B. Chen, F. Yi, and S. Zou, "Optimization of extraction of polysaccharide from dandelion root by response surface methodology: structural characterization and antioxidant activity," International Journal of Biological Macromolecules, vol. 140, pp. 907-919, 2019.

[46] X. Wang, M. Huang, F. Yang et al., "Rapeseed polysaccharides as prebiotics on growth and acidifying activity of probiotics in vitro," Carbohydrate Polymers, vol. 125, pp. 232-240, 2015.

[47] S. He, X. Wang, Y. Zhang et al., "Isolation and prebiotic activity of water-soluble polysaccharides fractions from the bamboo shoots (Phyllostachys praecox)," Carbohydrate Polymers, vol. 151, pp. 295-304, 2016.

[48] F. Huang, H. Liu, R. Zhang et al., "Physicochemical properties and prebiotic activities of polysaccharides from longan pulp based on different extraction techniques," Carbohydrate Polymers, vol. 206, pp. 344-351, 2019. 